\title{
DIGIT survey of far-infrared lines from protoplanetary discs
}

\author{
II. $\mathrm{CO}^{\star, \star \star}$ \\ Gwendolyn Meeus ${ }^{1}$, Colette Salyk ${ }^{2}$, Simon Bruderer ${ }^{3,4}$, Davide Fedele ${ }^{3}$, Koen Maaskant ${ }^{4}$, Neal J. Evans II $^{5}$, \\ Ewine F. van Dishoeck ${ }^{3,4}$, Benjamin Montesinos ${ }^{6}$, Greg Herczeg ${ }^{7}$, Jeroen Bouwman ${ }^{8}$, Joel D. Green ${ }^{5}$, \\ Carsten Dominik ${ }^{9,10}$, Thomas Henning ${ }^{8}$, Silvia Vicente ${ }^{11}$, and the DIGIT team \\ 1 Universidad Autónoma de Madrid, Dpto. Física Teórica, Fac. de Ciencias, Campus de Cantoblanco, 28049 Madrid, Spain \\ e-mail: gwendolyn.meeus@uam.es \\ 2 National Optical Astronomy Observatory, 950 N. Cherry Avenue, Tucson AZ 85719, USA \\ 3 Max Planck Institut für Extraterrestriche Physik, Giessenbachstrasse 1, 85748 Garching, Germany \\ ${ }^{4}$ Leiden Observatory, Leiden University, PO Box 9513, 2300 RA Leiden, The Netherlands \\ 5 The University of Texas at Austin, Department of Astronomy, 2515 Speedway, Stop C1400, Austin TX 78712-1205, USA \\ 6 Dept. of Astrophysics, CAB (CSIC-INTA), ESAC Campus, PO Box 78, 28691 Villanueva de la Cañada, Spain \\ Kavli Institute for Astronomy and Astrophysics, Yi He Yuan Lu 5, 100871 Beijing, PR China \\ 8 Max Planck Institute for Astronomy, Königstuhl 17, 69117 Heidelberg, Germany \\ 9 Anton Pannekoek Astronomical Institute, University of Amsterdam, PO Box 94249, 1090 GE Amsterdam, The Netherlands \\ 10 Department of Astrophysics/IMAPP, Radboud University Nijmegen, PO Box 9010, 6500 GL Nijmegen, The Netherlands \\ 11 Kapteyn Astronomical Institute, Postbus 800, 9700 AV Groningen, The Netherlands
}

Received 17 January 2013 / Accepted 12 August 2013

ABSTRACT

\begin{abstract}
$\mathrm{CO}$ is an important component of a protoplanetary disc as it is one of the most abundant gas phase species. Furthermore, observations of CO transitions can be used as a diagnostic of the gas, tracing conditions in both the inner and outer disc. We present Herschel/PACS spectroscopy of a sample of $22 \mathrm{Herbig} \mathrm{Ae/Be} \mathrm{(HAEBEs)} \mathrm{and} \mathrm{eight} \mathrm{T} \mathrm{Tauri} \mathrm{stars} \mathrm{(TTS),} \mathrm{covering} \mathrm{the} \mathrm{pure} \mathrm{rotational} \mathrm{CO} \mathrm{transitions}$ from $J=14 \rightarrow 13$ up to $J=49 \rightarrow 48$. CO is detected in only five HAEBEs, namely AB Aur, HD 36112, HD 97048, HD 100546, and IRS 48, and in four TTS, namely AS 205, S CrA, RU Lup, and DG Tau. The highest transition detected is $J=36 \rightarrow 35$ with $E_{\text {up }}$ of $3669 \mathrm{~K}$, seen in HD 100546 and DG Tau. We construct rotational diagrams for the discs with at least three CO detections to derive $T_{\text {rot }}$ and find average temperatures of $270 \mathrm{~K}$ for the HAEBEs and $485 \mathrm{~K}$ for the TTS. The HD 100546 star requires an extra temperature component at $T_{\text {rot }} \sim 900-1000 \mathrm{~K}$, suggesting a range of temperatures in its disc atmosphere, which is consistent with thermo-chemical disc models. In HAEBEs, the objects with $\mathrm{CO}$ detections all have flared discs in which the gas and dust are thermally decoupled. We use a small model grid to analyse our observations and find that an increased amount of flaring means higher line flux, as it increases the mass in warm gas. $\mathrm{CO}$ is not detected in our flat discs as the emission is below the detection limit. We find that HAEBE sources with $\mathrm{CO}$ detections have high $L_{\mathrm{UV}}$ and strong PAH emission, which is again connected to the heating of the gas. In TTS, the objects with $\mathrm{CO}$ detections are all sources with evidence of a disc wind or outflow. For both groups of objects, sources with $\mathrm{CO}$ detections generally have high UV luminosity (either stellar in HAEBEs or due to accretion in TTS), but this is not a sufficient condition for the detection of the far-IR CO lines.
\end{abstract}

Key words. circumstellar matter - protoplanetary disks

\section{Introduction}

It is firmly established that circumstellar discs around young stars are sites of planet formation. During the first $10 \mathrm{Myr}$, the initial gas-rich disc first evolves into a transitional and then finally into a debris disc while dispersing its gas content. The knowledge of this dispersal process and its properties is a crucial part of the planet-formation puzzle, as the amount of gas present in a disc determines whether gas-rich planets can form. Therefore, three aspects need to be characterised well: the disc geometry, the solid state content, and the gas content. Both the geometry and the dust in Herbig Ae/Be (HAEBE) discs are

\footnotetext{
* Herschel is an ESA space observatory with science instruments provided by European-led Principal Investigator consortia and with important participation from NASA.

$\star \star$ Appendices are available in electronic form at http://www. aanda.org
}

well-studied (e.g., the review by Williams \& Cieza 2011; Meeus et al. 2001; Dominik et al. 2003; and Benisty et al. 2010 for the geometry; Acke et al. 2010 for the polycyclic aromatic hydrocarbon (PAH); and Juhász et al. 2010 for the dust properties).

To understand the spatial distribution, chemistry and physical properties of the gas component it is necessary to observe a range of transitions in several species, as they can originate in different regions of the disc under distinct conditions (density, temperature, and radiation field). For the purpose of studying the gas disc, $\mathrm{H}_{2}$ and $\mathrm{CO}$ lines are most often used, since they are the most abundant molecules present. In the FUV, $\mathrm{H}_{2}$ probes the warm gas and is frequently detected in T Tauri stars (TTS; e.g., Herczeg et al. 2006; Ingleby et al. 2011; France et al. 2012). In the IR, the detection of $\mathrm{H}_{2}$ is more difficult, due to the nature of the $\mathrm{H}_{2}$ molecule (homonuclear with wide spacing of energy levels). In a survey of 15 HAEBEs with CRIRES, Carmona et al. (2011) have detected ro-vibrational transitions at $2.1218 \mu \mathrm{m}$ in 
only two objects. Earlier, Bitner et al. (2008), Carmona et al. (2008), and Martin-Zaïdi et al. (2009, 2010) searched for pure rotational lines of $\mathrm{H}_{2}$ at $17.035 \mu \mathrm{m}$ in a sample of 20 HAEBEs; only two detections were reported. In TTS, the success rate is a little higher: Lahuis et al. (2007) have detected mid-IR $\mathrm{H}_{2}$ emission lines in six TTS, while Bary et al. (2008) have found five more $\mathrm{H}_{2}$ detections in the near-IR.

In sharp contrast, the detection of $\mathrm{CO}$, although less abundant, is much easier. Its lines have been used to trace both the inner and outer disc. The fundamental ro-vibrational $\mathrm{CO}$ band $(\Delta v=1)$ at $4.7 \mu \mathrm{m}$ traces warm $(T>100 \mathrm{~K})$ gas in the terrestrial planet-forming region (0.1-10 AU). This band is often observed in HAEBEs (e.g., Blake \& Boogert 2004; Brittain et al. 2007) and in TTS (e.g., Najita et al. 2003; Salyk et al. 2011). The bands are rotationally excited up to high $J(>30)$, resulting in a $T_{\text {rot }}$ between 900 and $2500 \mathrm{~K}$. The rotational temperature is much lower for ${ }^{13} \mathrm{CO}$ at $\sim 250 \mathrm{~K}$ (Brown et al. 2013). UV fluorescence can cause super-thermal level populations, as observed (Brittain et al. 2007) and modelled (Thi et al. 2013) in UV-bright HAEBEs, where $T_{\text {vib }}>5000 \mathrm{~K}$, and in TTS (Bast et al. 2011; Brown et al. 2013). While some HAEBEs have CO extending to the dust sublimation radius (Salyk et al. 2011), others have evidence of significant inner disc gas clearing (Goto et al. 2006; Brittain et al. 2007; Pontoppidan et al. 2008; van der Plas et al. 2009). Thi et al. (2013), and van der Plas (2010) find that $T_{\text {rot }}<$ $T_{\text {vib }}$ in flaring discs and the inner radius of $\mathrm{CO}$ is $r_{\text {in }} \sim 10 \mathrm{AU}$, while $T_{\text {vib }} \lesssim T_{\text {rot }}$ in self-shadowed discs, and $r_{\text {in }} \sim 1$ AU: flaring HAEBE discs thus have lower $\mathrm{CO}$ abundances in their inner regions than flat discs. In a recent paper, Maaskant et al. (2013) show that several group I sources (the flaring discs) have dustdepleted gaps separating an optically thin inner disc and a flaring outer disc. It has been proposed that dust-depleted regions are a typical property of group I discs. This geometry allows more radiation to reach the outer disc, so they can be heated more when compared to the group II (flat discs). For the TTS, Brown et al. (2013) have found that most of the line profiles in TTS indicate that the $\mathrm{CO}$ emission originates in the disc with a slow disc wind.

On the other end of the spectrum, CO low- $J$ pure rotational transitions $(\Delta v=0)$ can be observed at millimeter wavelengths. These low- $J$ transitions originate in cold, optically thick CO that is located in the outer disc and are routinely detected in HAEBEs (e.g., Thi et al. 2001; Dent et al. 2005) and in TTS (e.g., Koerner \& Sargent 1995; Öberg et al. 2010). As the lines are optically thick, the outer disc radius can be estimated from the line profile (e.g., Dent et al. 2005; Panić et al. 2008). In HAEBE discs, the dust temperature in the entire disc is high enough (at least $30 \mathrm{~K}$ ) to avoid freeze-out of $\mathrm{CO}$ on the dust grains (e.g., Panic et al. 2009; Bruderer et al. 2012), while this happens more frequently in TTS (e.g., Hersant et al. 2009).

The much less observed far-IR wavelength range covers rotationally-excited mid- to high- $J$ CO lines, which probe intermediate temperatures between the low- $J$ sub-mm and the vibrationally-excited near-IR lines. Giannini et al. (1999) analysed ISO/LWS observations of 3 HAEBEs, covering rotational lines from $J_{\text {up }}=14$ to $J_{\text {up }}=19$. Their modelling showed that the $\mathrm{CO}$ emission originates in a compact region (up to a few $100 \mathrm{AU}$ ) around the central star and suggest that the stellar FUV radiation excites the $\mathrm{CO}$.

The far-IR lines of $\mathrm{CO}$ in HAEBEs and TTS were also observed by DIGIT (Sturm et al. 2010) and GASPS (Meeus et al. 2012) with the instrument PACS (Poglitsch et al. 2010) onboard Herschel (Pilbratt et al. 2010). Bruderer et al. (2012; hereafter BR12) have modelled pure rotational CO transitions in HD 100546 (Sturm et al. 2010). They have showed the following: 1) On the disc surface, the gas temperature is decoupled from the dust temperature. 2) The low- $J(J<10)$ lines trace a radius between $70-220 \mathrm{AU}$ at a low scale-height in the disc. The mid- $J(J<20)$ lines trace a radius of 35-80 AU, while the high- $J(J>20)$ lines trace the upper disc atmosphere at 20 $50 \mathrm{AU}$. Therefore, the higher the $J$, the closer the star is to the region that the transition traces. 3) The highest $J$ lines are optically thin and scale with the amount of hot gas in the disc surface, while the lowest $J$ lines are optically thick and scale with the outer disc radius. The authors also show that the rotational diagram of HD 100546 (including also APEX data of low- $J$ transitions) can be fitted with three temperature components: $T_{\text {rot }} \sim$ 60,300 , and $750 \mathrm{~K}$

We now present PACS spectra of a much larger sample of HAEBEs and TTS that was observed within the DIGIT Open Time Key Project (OTKP; P.I. Neal Evans). These spectra show pure rotational transitions of CO with $J_{\text {up }}$ from 14 to 49 , corresponding to $E_{\text {up }}$ between 581 and $6724 \mathrm{~K}$. Detections of O I, C II, $\mathrm{OH}, \mathrm{H}_{2} \mathrm{O}$, and $\mathrm{CH}^{+}$are reported in a companion paper by Fedele et al. (2013; Paper I). In Sect. 2, we present the sample and the observations, while we show the line detections and fluxes and construct rotational diagrams in Sect. 3. In Sect. 4, we look into the UV luminosity and use a small disc model grid to explain our observations. In Sect. 5, we speculate on the impact of PAH luminosity and inner disc clearing on the $\mathrm{CO}$ emission, while we present our conclusions in Sect. 6.

\section{Sample and observations}

Our sample consists of 22 HAEBEs and eight TTS. The HAEBE sample has spectral types between B9 and F4 and is representative for the HAe stars, while the TTS sources tend to be distinct from typical TTS in some way (such as having a large amount of veiling, or the presence of a strong disc wind, outflow, or a disc gap). We refer to Paper I for a discussion of the sample properties.

For each HAEBE star, we derived its disc group (I: flared discs, II: flat discs, in the classification of Meeus et al. 2001) and calculated $L_{\mathrm{UV}}$ by integrating the UV flux obtained from the IUE archive between 1150 and $2430 \AA$ for the HAEBEs. For the TTS, we measured $L_{\mathrm{UV}}$ between 1250-1700 $\AA$ directly for a few sources. When direct measurements of FUV were not available, they were derived from observations of the $\mathrm{H}_{\mathrm{I}} \operatorname{Pf} \beta$ line and from an empirical relationship between $L_{\text {acc }}$ and $\operatorname{Pf} \beta$ (Salyk et al. 2013 ) in conjunction with an empirical relationship between $L_{\text {acc }}$ and $L_{\mathrm{UV}}$ (Yang et al. 2012). These results are listed in Table 1.

We obtained Herschel/PACS spectroscopy in SED range mode, covering the spectral range between 51 and $210 \mu \mathrm{m}$. Not all sources were observed in the full range. For an overview of the observational settings and the reduction methods, we refer to Paper I, where we also show the full range spectra for each object. In Fig. 1 we zoom in at the positions of the CO lines for HD 100546, while we show similar plots in Figs. A.1 to A.8 for those objects where CO was detected.

With the sensitivity of our spectra, we are able to achieve a typical signal-to-noise ratio $(\mathrm{S} / \mathrm{N})$ on the continuum of 100 and to detect a line with $3 \sigma$ confidence when the line flux is higher than $1 \times 10^{-17} \mathrm{~W} \mathrm{~m}^{-2}$, which varies with the observed wavelength and level of continuum flux. Furthermore, the spectra become more noisy at the edges of the bands, resulting in higher upper limits (for a more detailed discussion on this topic, we refer to the discussion in the DIGIT data paper by Green et al. 2013). For detected lines, we extracted the line fluxes using a Gaussian fit to the emission lines by using the rms on the 
Table 1. Some properties of the sample.

\begin{tabular}{|c|c|c|c|c|}
\hline HAEBE & $\begin{array}{l}\text { Disc } \\
\text { group }\end{array}$ & $\begin{array}{r}L_{\mathrm{UV}} / L_{\odot} \\
1150-2430 \AA\end{array}$ & $\begin{array}{l}L_{\mathrm{PAH}} \\
\left(L_{\odot}\right)\end{array}$ & $\begin{array}{l}\mathrm{CO} \\
\text { det. }\end{array}$ \\
\hline AB Aur & I & 4.63 & 0.203 & $\mathrm{Y}$ \\
\hline HD 35187 & II & 2.23 & 0.034 & $\mathrm{n}$ \\
\hline HD 36112 & I & 1.32 & 0.029 & Y \\
\hline HD 38120 & I & - & 0.116 & $\mathrm{n}$ \\
\hline HD 50138 & II & - & - & $\mathrm{n}$ \\
\hline HD 97048 & I & 7.69 & 0.367 & Y \\
\hline HD 98922 & II & - & - & $\mathrm{n}$ \\
\hline HD 100453 & I & 0.29 & 0.038 & $\mathrm{n}$ \\
\hline HD 100546 & I & 7.22 & 0.098 & $\mathrm{Y}$ \\
\hline HD 104237 & II & 1.54 & - & $\mathrm{n}$ \\
\hline HD 135344 B & I & $>0.11^{\mathrm{a}}$ & 0.015 & $\mathrm{n}$ \\
\hline HD 139614 & I & 0.39 & 0.022 & $\mathrm{n}$ \\
\hline HD 141569 A & II/TO & 6.83 & 0.007 & $\mathrm{n}$ \\
\hline HD 142527 & I & $>0.15^{\mathrm{a}}$ & 0.149 & $\mathrm{n}$ \\
\hline HD 142666 & II & $0.37-0.68$ & 0.028 & $\mathrm{n}$ \\
\hline HD 144432 & II & - & 0.003 & $\mathrm{n}$ \\
\hline HD 144668 & II & $1.55-2.94$ & - & $\mathrm{n}$ \\
\hline IRS 48 & I & 4.63 & 0.386 & $\mathrm{Y}$ \\
\hline HD 150193 & II & 8.53 & - & $\mathrm{n}$ \\
\hline HD 163296 & II & $3.21-5.58$ & - & $\mathrm{n}$ \\
\hline HD 169142 & I & 0.45 & 0.093 & $\mathrm{n}$ \\
\hline HD 179218 & I & - & - & $\mathrm{n}$ \\
\hline TTS & $\log L_{\text {acc }} / L_{\odot}$ & $\begin{array}{r}L_{\mathrm{UV}} / L_{\odot} \\
1250-1700 \AA\end{array}$ & $\begin{array}{l}\mathrm{CO} \\
\text { det. }\end{array}$ & \\
\hline DG Tau & -0.13 & $0.016^{b}$ & $\mathrm{Y}$ & \\
\hline HT Lup & -0.65 & $0.006^{b}$ & $\mathrm{n}$ & \\
\hline RU Lup & -0.42 & 0.01 & $\mathrm{Y}$ & \\
\hline RY Lup & - & 0.002 & $\mathrm{n}$ & \\
\hline AS $205 \mathrm{~A} / \mathrm{B}$ & $-0.15 /-0.37$ & $0.016 / 0.01$ & Y & \\
\hline SR 21 & - & $0.0003^{b}$ & $\mathrm{n}$ & \\
\hline RNO90 & -0.16 & $0.016^{b}$ & $\mathrm{n}$ & \\
\hline $\mathrm{S} \mathrm{CrA} \mathrm{A/B}$ & $-0.10 /-0.59$ & $0.016 / 0.006^{b}$ & $\mathrm{Y}$ & \\
\hline
\end{tabular}

Notes. HAEBE disc group classification from Meeus et al. (2001), Acke et al. (2010), and Maaskant et al. (2013). "TO" stands for "Transition Object", indicating that it lacks a near-IR excess. $L_{\mathrm{UV}}$ is determined differently for the HAEBEs and the TTS, as described in the text (see Sect. 2); (a) $L_{\mathrm{UV}}$ measured on the model photosphere (no UV observations available); ${ }^{(b)} L_{\mathrm{UV}}$ estimated from accretion luminosities. $L_{\mathrm{PAH}}$ is from the survey by Acke et al. (2010) which includes our HAEBE sample; with "-", we indicate a non-detection. In the column "CO det.", we indicate whether there was CO emission detected in our PACS spectra (Y) or not (n).

continuum (excluding the line) to derive a $1 \sigma$ error on the line flux. For non-detections, we give a $3 \sigma$ upper limit, which is also calculated from the continuum rms. The derived line fluxes are listed in Table 2. The second row for each source is the flux density in the adjacent continuum.

\section{Results}

\subsection{Detections}

We detected CO lines in only 5 HAEBE stars of our sample: AB Aur, HD 36112, HD 97048, HD 100546, and IRS 48. While all 5 detections toward HAEBEs occur for fairly nearby stars $\langle d\rangle=159 \pm 71 \mathrm{pc}$, there are many HAEBEs at similar distances without detections. All 5 HAEBEs with detections have group I (flaring) discs; there is no $\mathrm{CO}$ detection seen in a group II (flat) HAEBE disc. Within the group I sources, the sources with CO detections have the highest $L_{\mathrm{UV}}$ observed.
For the TTS, we detected CO in 4 objects: AS 205, a disc wind source (Bast et al. 2011) and a possible outflow source (Mundt 1984); S CrA and B, which are disc wind sources (Bast et al. 2011); RU Lup, which is a disc wind source (Bast et al. 2011) and an outflow source (Herczeg et al. 2005, and references therein); and DG Tau, which is an outflow source (e.g., Lavalley-Fouquet et al. 2000). An equivalent disc geometry classification for the TTS does not exist, so we could not compare the two samples in this regard. However, $\mathrm{CO}$ detections are only seen in TTS that show evidence of a strong disc wind (according to the line shape criterion defined in Bast et al. 2011), a resolved outflow, or both. It is not yet known whether there is a correlation between the presence of disc winds and outflows, although several discs have signatures of both.

The highest observable transition is $J=49 \rightarrow 48$, but the highest detected transition in our spectra is $J=36 \rightarrow 35$, as seen only in HD 100546 and in the outflow source DG Tau. In Table A.1, we list the transitions, their wavelength and the upper level energy (in kelvin) that are detected in our spectra. The transition $J=31 \rightarrow 30$ is at the same wavelength as an $\mathrm{OH}$ line; therefore, we consider it to be an upper limit in our analysis.

\subsection{Rotation diagrams}

Since we covered a wide range in transitions, it is possible to analyse our data with the aid of rotational diagrams (see Goldsmith \& Langer 1999). We constructed rotational diagrams for the sources with at least three $\mathrm{CO}$ line detections (see Fig. 2) and list the derived $T_{\text {rot }}$ in Table 3 . We derived the error on $T_{\text {rot }}$ by calculating the 2 extreme temperatures that are still consistent with the errors on the line fluxes. We define the $Y$ axis of the diagram as $\ln \left(\mathrm{d} \Omega N_{\mathrm{u}} / g_{\mathrm{u}}\right)=\ln \left(4 \pi F_{\mathrm{u}} /\left(A_{\mathrm{u}} h v g_{\mathrm{u}}\right)\right)$ to include the solid angle $\Omega$ of the emission, since the CO ladder could emerge from different regions in the disc (see discussion in BR12). For the HAEBEs, we obtain $T_{\text {rot }}$ between 200 and $350 \mathrm{~K}$. For HD 100546, we also need a second component to fit the rotational diagram with $T_{\text {rot }} \sim 900-1000 \mathrm{~K}$. A second component could also be present in AB Aur and HD 97048 but we do not have enough line detections to derive a temperature, in these cases. More sensitive data could reveal higher $J$ emission lines also for these sources. The result for HD 100546 is similar to that found in an earlier analysis (Sturm et al. 2010). We note that the fit predicts line fluxes that are too high when compared to the observations for a few transitions (around $22 \rightarrow$ 21 , or $\left.E_{\mathrm{up}}=1397 \mathrm{~K}\right)$. It is not clear whether to attribute this to a bad flux calibration of this wavelength range $(\sim 118 \mu \mathrm{m})$ and/or to the low quality of this spectral region. The mean of the HAEBE $T_{\text {rot }}$ values, excluding the hot component in HD 100546 , is $\left\langle T_{\text {rot }}\right\rangle=271 \pm 39 \mathrm{~K}$. For TTS, the temperatures are higher, ranging between 350 and $600 \mathrm{~K}$ with the outflow source DG Tau having the highest $T_{\text {rot }}$ at a value of $582 \pm 12 \mathrm{~K}$. The mean value is $486 \pm 104 \mathrm{~K}$, which is significantly higher than the mean for the HAEBEs.

The rotational temperatures of $200-350 \mathrm{~K}$ in HAEBE discs are lower than those typically found from ${ }^{12} \mathrm{CO}$ ro-vibrational fundamental $(v=1 \rightarrow 0)$ near-IR transitions (Salyk et al. 2011). They are comparable, however, to the rotational temperatures derived from the ${ }^{13} \mathrm{CO}$ ro-vibrational fundamental lines, which probe distances somewhat further out into the disc (Brown et al. 2013).

We note that converting $T_{\text {rot }}$ derived from a rotation diagram to a kinetic temperature is not straightforward, as it assumes optically thin emission. Even if the lines are thin, $T_{\text {rot }}$ equals $T_{\text {gas }}$ only if the excitation is in thermal equilibrium with the gas, 

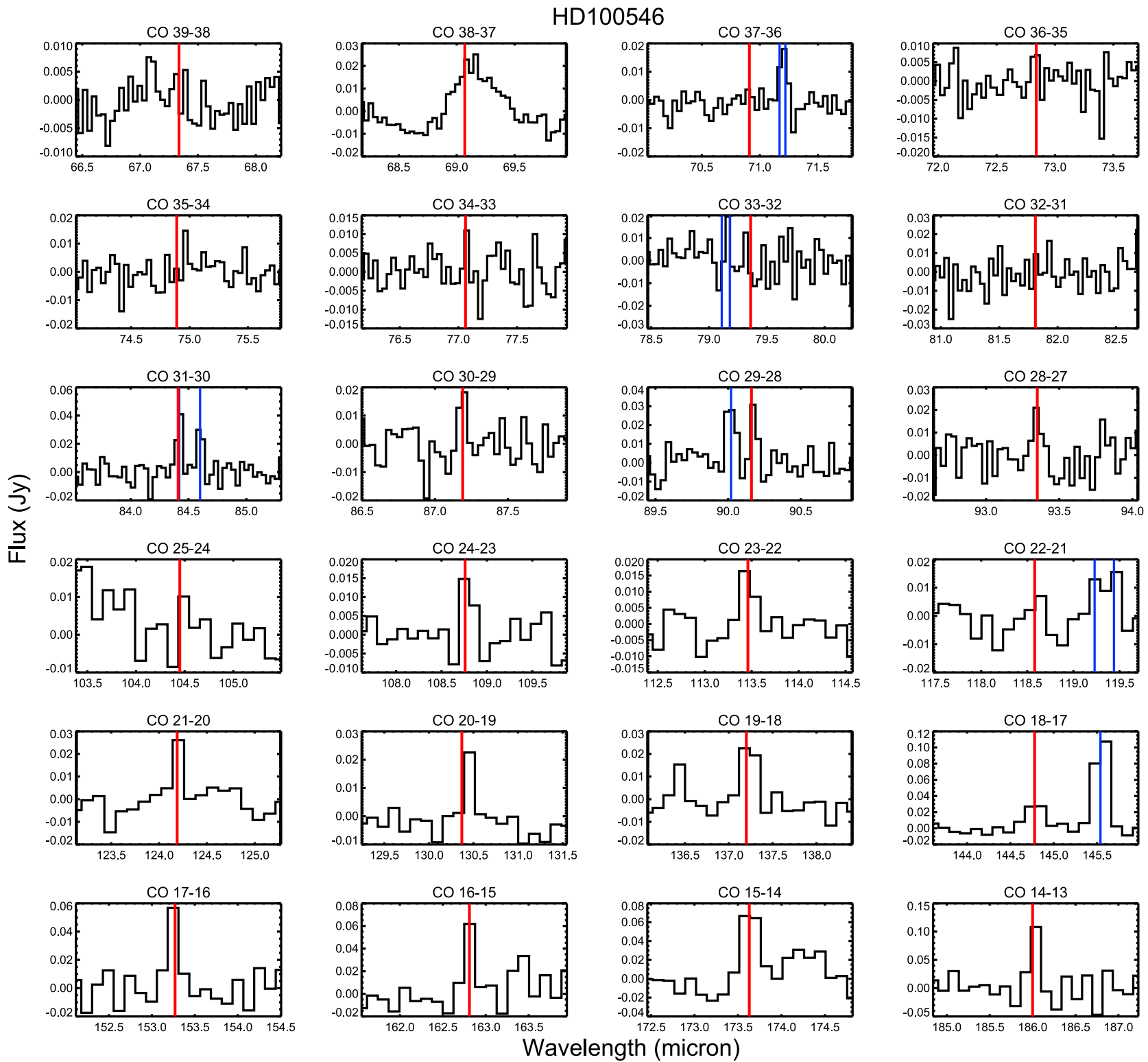

Fig. 1. Spectra of HD 100546, centered on the CO lines, indicated in red. The OH line at $84.42 \mu \mathrm{m}$ blends with the CO $J=31 \rightarrow 30$ line. In blue, we indicate the positions of other lines $\left(\mathrm{CH}^{+}\right.$at $90 \mu \mathrm{m}$ and $\left[\mathrm{O}_{\mathrm{I}}\right]$ at $145 \mu \mathrm{m}$; rest are $\mathrm{OH}$ doublets).

i.e., if the density is high enough. Indeed, Bruderer et al. (2012) discussed that the $\mathrm{CO}$ ladder reflects emission from the disc atmosphere from a range of radii ( $\sim 20-80 \mathrm{AU})$ at different temperatures and that the shape of the CO ladder can depend on several factors: 1) the gas temperature and density; 2) optical depth effects for the low- $J$ to mid- $J$ lines; and 3 ) either the amount of emitting molecules if the line is optically thin, or the size of the emitting region if the line is optically thick. For HD 100546, Bruderer et al. (2012) predicted that lines up to $J=16 \rightarrow 15$ are optically thick. Assuming that the model for HD 100546 is relevant for all our sources, the lines we observe are mainly optically thin so that the gas temperature and density are the main factors, assuming a normal $\mathrm{CO}$ abundance.

\section{Analysis}

\subsection{Relation with the (UV) luminosity}

Does the far-IR CO line emission depend on stellar luminosities? We compared average HAEBE and TTS CO line luminosities for the $J=17 \rightarrow 16$ transition and found that they are remarkably similar: we find $(2.06 \pm 0.7) \times 10^{-5} L_{\odot}$ for the HAEBEs and $(2.06 \pm 1.0) \times 10^{-5} L_{\odot}$ for the TTS, while the average bolometric luminosities are 86.0 $L_{\odot}$ and 5.0 $L_{\odot}$ for the HAEBEs and TTS, respectively. This indicates that there is no correlation between the average line luminosity and $L_{\mathrm{bol}}$.

But what about the UV luminosity? Models suggest that UV radiation is important for the gas heating and the excitation of the molecules and is one of the main factors determining the strength of the emission lines observed in the far-IR (e.g., Woitke et al. 2010; Kamp et al. 2011). Also, BR12 showed the importance of the temperature of the stellar radiation field with respect to the amount of line flux (see their Fig. 13). Because of self-shielding, the $\mathrm{CO}$ molecule can survive the UV radiation in the surface layers, and the $\mathrm{CO}$ emission lines can trace the heated disc surface. Since CO is an important coolant, an increased amount of heating leads to the detection of higher $J$ lines, and a higher line luminosity.

The HAEBE stars with strong $L_{\mathrm{UV}}$ and flaring discs (group I) often have a high [O I] $63 \mu \mathrm{m}$ line flux (Meeus et al. 2012; 
Table 2. Observed CO line fluxes in $\mathrm{W} \mathrm{m}^{-2} \times 10^{-18}$ (first row) and adjacent continuum flux density in Jy (second row).

\begin{tabular}{|c|c|c|c|c|c|c|c|c|}
\hline Transition & $J=38 \rightarrow 37$ & $J=37 \rightarrow 36$ & $J=36 \rightarrow 35$ & $J=35 \rightarrow 34$ & $J=34 \rightarrow 33$ & $J=33 \rightarrow 32$ & $J=32 \rightarrow 31$ & $J=31 \rightarrow 30^{a}$ \\
\hline \multirow[t]{2}{*}{ AB Aur } & $<100.5$ & $<95.3$ & $<100.7$ & $<73.8$ & $<100.3$ & $<79.5$ & $<77.9$ & $92.1(2.6)$ \\
\hline & $134.8 \mathrm{Jy}$ & $134.3 \mathrm{Jy}$ & $135.5 \mathrm{Jy}$ & $136.6 \mathrm{Jy}$ & $134.8 \mathrm{Jy}$ & $133.1 \mathrm{Jy}$ & $129.7 \mathrm{Jy}$ & $132.0 \mathrm{Jy}$ \\
\hline \multirow[t]{2}{*}{ HD 36112} & $<21.8$ & $<25.2$ & $<38.4$ & $<24.3$ & $<20.3$ & $<25.1$ & $<24.9$ & $<29.5$ \\
\hline & $20.5 \mathrm{Jy}$ & $20.5 \mathrm{Jy}$ & $20.6 \mathrm{Jy}$ & $20.3 \mathrm{Jy}$ & $20.3 \mathrm{Jy}$ & $20.0 \mathrm{Jy}$ & $19.1 \mathrm{Jy}$ & $19.1 \mathrm{Jy}$ \\
\hline \multirow[t]{2}{*}{ HD 97048} & $<42.8$ & $<45.7$ & $<38.2$ & $<33.1$ & $<42.4$ & $<37.6$ & $<40.2$ & $56.6(15.0)$ \\
\hline & $65.8 \mathrm{Jy}$ & $66.6 \mathrm{Jy}$ & $68.1 \mathrm{Jy}$ & $68.5 \mathrm{Jy}$ & 70.3Jy & 69.1 Jy & $66.0 \mathrm{Jy}$ & $68.5 \mathrm{Jy}$ \\
\hline \multirow[t]{2}{*}{ HD 100546} & $<107.4$ & $<82.9$ & $52.9(12.6)$ & $<44.1$ & $53.1(14.4)$ & $<84.5$ & $<65.3$ & $139.7(27.6)$ \\
\hline & $172.0 \mathrm{Jy}$ & $162.4 \mathrm{Jy}$ & $160.6 \mathrm{Jy}$ & $159.5 \mathrm{Jy}$ & $156.7 \mathrm{Jy}$ & $148.6 \mathrm{Jy}$ & 139.7 Jy & $141.7 \mathrm{Jy}$ \\
\hline \multirow[t]{2}{*}{ IRS 48} & $<28.7$ & $<23.0$ & $<37.5$ & $<31.2$ & $<28.4$ & $<37.9$ & $<38.6$ & $<33.7$ \\
\hline & $48.9 \mathrm{Jy}$ & $47.2 \mathrm{Jy}$ & 46.4 Jy & $46.6 \mathrm{Jy}$ & $45.1 \mathrm{Jy}$ & $42.6 \mathrm{Jy}$ & $39.8 \mathrm{Jy}$ & $39.4 \mathrm{Jy}$ \\
\hline \multirow[t]{2}{*}{ AS 205} & $<22.8$ & $<33.1$ & $<37.0$ & $<29.8$ & $<24.1$ & $<37.2$ & $<25.9$ & $46.0^{\circ}$ \\
\hline & $20.7 \mathrm{Jy}$ & $20.5 \mathrm{Jy}$ & $21.0 \mathrm{Jy}$ & $20.8 \mathrm{Jy}$ & $20.9 \mathrm{Jy}$ & $20.7 \mathrm{Jy}$ & $19.3 \mathrm{Jy}$ & $20.4 \mathrm{Jy}$ \\
\hline \multirow[t]{2}{*}{$\mathrm{S} \mathrm{CrA}$} & $<24.0$ & $<33.2$ & $<37.9$ & $<28.3$ & $<30.4$ & $<42.6$ & $<30.8$ & $<40.8$ \\
\hline & $23.6 \mathrm{Jy}$ & $24.0 \mathrm{Jy}$ & $24.5 \mathrm{Jy}$ & $24.3 \mathrm{Jy}$ & $24.6 \mathrm{Jy}$ & $24.4 \mathrm{Jy}$ & $23.7 \mathrm{Jy}$ & 24.5 \\
\hline \multirow[t]{3}{*}{ DG Tau } & $<15.4$ & $<42.4$ & 25.7 (6.6) & $<18.9$ & $24.7(5.5)$ & $<47.6$ & 19.8: (7.0) & $128.3(25.3)$ \\
\hline & $26.5 \mathrm{Jy}$ & $26.6 \mathrm{Jy}$ & $26.7 \mathrm{Jy}$ & $26.6 \mathrm{Jy}$ & $26.7 \mathrm{Jy}$ & $26.1 \mathrm{Jy}$ & $25.2 \mathrm{Jy}$ & $26.2 \mathrm{Jy}$ \\
\hline & $J=30 \rightarrow 29$ & $J=29 \rightarrow 28$ & $J=28 \rightarrow 27$ & $J=25 \rightarrow 24$ & $J=24 \rightarrow 23$ & $J=23 \rightarrow 22$ & $J=22 \rightarrow 21$ & \\
\hline \multirow[t]{2}{*}{ AB Aur } & $<57.8$ & $<81.5$ & $<55.5$ & $<54.2$ & $<47.0$ & $58.9(12.4)$ & $<45.7$ & \\
\hline & $133.0 \mathrm{Jy}$ & $129.8 \mathrm{Jy}$ & 123.6Jy & $103.7 \mathrm{Jy}$ & $99.2 \mathrm{Jy}$ & $93.8 \mathrm{Jy}$ & $88.0 \mathrm{Jy}$ & \\
\hline \multirow[t]{2}{*}{ HD 36112} & $<27.6$ & $<22.7$ & $<32.3$ & $<17.7$ & $<13.3$ & $<11.0$ & $<9.1$ & \\
\hline & $19.7 \mathrm{Jy}$ & $19.4 \mathrm{Jy}$ & $19.0 \mathrm{Jy}$ & $15.9 \mathrm{Jy}$ & $15.5 \mathrm{Jy}$ & $14.6 \mathrm{Jy}$ & $13.7 \mathrm{Jy}$ & \\
\hline \multirow[t]{2}{*}{ HD 97048} & $<43.0$ & $<32.5$ & $<42.7$ & $<36.4$ & $<30.4$ & $30.1(8.7)$ & 14.7: (6.6) & \\
\hline & $71.1 \mathrm{Jy}$ & $70.7 \mathrm{Jy}$ & $68.2 \mathrm{Jy}$ & $61.5 \mathrm{Jy}$ & $59.1 \mathrm{Jy}$ & $57.4 \mathrm{Jy}$ & $53.7 \mathrm{Jy}$ & \\
\hline \multirow[t]{2}{*}{ HD 100546} & $73.4(14.9)$ & $78.6(18.7)$ & $81.5(11.2)$ & $<77.1$ & $71.3(11.5)$ & $78.4(11.2)$ & $<42.0$ & \\
\hline & $139.6 \mathrm{Jy}$ & $135.6 \mathrm{Jy}$ & $126.1 \mathrm{Jy}$ & $107.9 \mathrm{Jy}$ & $100.5 \mathrm{Jy}$ & $91.5 \mathrm{Jy}$ & $83.2 \mathrm{Jy}$ & \\
\hline \multirow[t]{2}{*}{ IRS 48} & $<26.0$ & $<29.0$ & $<27.3$ & $<22.7$ & $<17.8$ & $<13.0$ & $<14.2$ & \\
\hline & $39.0 \mathrm{Jy}$ & $36.8 \mathrm{Jy}$ & $34.4 \mathrm{Jy}$ & $28.3 \mathrm{Jy}$ & $25.7 \mathrm{Jy}$ & $23.2 \mathrm{Jy}$ & $20.8 \mathrm{Jy}$ & \\
\hline \multirow[t]{2}{*}{ AS 205} & $<28.3$ & $<20.8$ & $55.1(7.3)$ & $18.9(5.4)$ & $15.2(4.8)$ & $30.1(3.7)$ & $17.9(3.8)$ & \\
\hline & $19.8 \mathrm{Jy}$ & 20.1 & $19.1 \mathrm{Jy}$ & $15.9 \mathrm{Jy}$ & $15.4 \mathrm{Jy}$ & $14.5 \mathrm{Jy}$ & 13.7 & \\
\hline \multirow[t]{2}{*}{$\mathrm{S} \mathrm{CrA}$} & $<24.6$ & $<23.2$ & $24.9(6.4)$ & $26.6(6.5)$ & $23.1(5.7)$ & $42.2(4.9)$ & $31.4(10.6)$ & \\
\hline & 24.4 Jy & $24.3 \mathrm{Jy}$ & $23.6 \mathrm{Jy}$ & $21.2 \mathrm{Jy}$ & 20.3 Jy & $19.0 \mathrm{Jy}$ & $17.6 \mathrm{Jy}$ & \\
\hline \multirow[t]{3}{*}{ DG Tau } & $36.4(6.2)$ & $41.3(7.3)$ & $51.5(6.9)$ & $39.8(4.8)$ & $38.4(4.1)$ & $64.9(3.3)$ & $40.2(8.4)$ & \\
\hline & $26.5 \mathrm{Jy}$ & $26.0 \mathrm{Jy}$ & $25.5 \mathrm{Jy}$ & $21.4 \mathrm{Jy}$ & $20.5 \mathrm{Jy}$ & $19.4 \mathrm{Jy}$ & $18.5 \mathrm{Jy}$ & \\
\hline & $J=21 \rightarrow 20$ & $J=20 \rightarrow 19$ & $J=19 \rightarrow 18$ & $J=18 \rightarrow 17$ & $J=17 \rightarrow 16$ & $J=16 \rightarrow 15$ & $J=15 \rightarrow 14$ & $J=14 \rightarrow 13$ \\
\hline \multirow[t]{2}{*}{ AB Aur } & $<35.0$ & $39.2(9.3)$ & $<29.8$ & $49.6(10.3)$ & $40.7(14.4)$ & $<27.0$ & $64.0(16.1)$ & $<30.1$ \\
\hline & 83.9 Jy & $80.5 \mathrm{Jy}$ & $72.7 \mathrm{Jy}$ & $73.5 \mathrm{Jy}$ & 64.9 & $63.8 \mathrm{Jy}$ & $50.6 \mathrm{Jy}$ & $38.0 \mathrm{Jy}$ \\
\hline \multirow[t]{2}{*}{ HD 36112} & $<8.4$ & $7.9(2.5)$ & $<8.2$ & $<12.4$ & $<11.4$ & $<12.8$ & $14.1(4.1)$ & $16.0(4.7)$ \\
\hline & $13.2 \mathrm{Jy}$ & $12.8 \mathrm{Jy}$ & $12.5 \mathrm{Jy}$ & $12.3 \mathrm{Jy}$ & $12.2 \mathrm{Jy}$ & $12.0 \mathrm{Jy}$ & $11.0 \mathrm{Jy}$ & $8.2 \mathrm{Jy}$ \\
\hline HD 97048 & $<15.3$ & $<21.5$ & $<19.5$ & $25.8(7.1)$ & $32.4(5.3)$ & $<24.5$ & $49.1(11.5)$ & $<23.8$ \\
\hline & $55.1 \mathrm{Jy}$ & $53.3 \mathrm{Jy}$ & $54.2 \mathrm{Jy}$ & $58.2 \mathrm{Jy}$ & $58.8 \mathrm{Jy}$ & $58.1 \mathrm{Jy}$ & $54.3 \mathrm{Jy}$ & $43.9 \mathrm{Jy}$ \\
\hline HD 100546 & $65.0(8.8)$ & $49.9(5.7)$ & $64.7(8.5)$ & $71.5(6.9)$ & $73.9(10.4)$ & $58.8(9.7)$ & $88.3(9.7)$ & $60.0(8.3)$ \\
\hline & $75.1 \mathrm{Jy}$ & $68.4 \mathrm{Jy}$ & $64.1 \mathrm{Jy}$ & $60.5 \mathrm{Jy}$ & $57.0 \mathrm{Jy}$ & $52.8 \mathrm{Jy}$ & $45.9 \mathrm{Jy}$ & $35.0 \mathrm{Jy}$ \\
\hline IRS 48 & $9.9(3.0)$ & $12.3(4.1)$ & $11.1(3.7)$ & $13.4(2.8)$ & $22.7(4.7)$ & 12.0: (4.8) & $<18.4$ & $<21.5$ \\
\hline & $18.7 \mathrm{Jy}$ & $17.0 \mathrm{Jy}$ & 15.7 Jy & $14.4 \mathrm{Jy}$ & $13.3 \mathrm{Jy}$ & $12.3 \mathrm{Jy}$ & $10.2 \mathrm{Jy}$ & $7.2 \mathrm{Jy}$ \\
\hline AS 205 & $22.1(2.3)$ & $20.3(2.8)$ & $21.6(2.4)$ & $18.5(2.6)$ & $18.9(3.9)$ & $13.0(3.8)$ & $24.0(5.5)$ & $16.3(4.2)$ \\
\hline & $13.1 \mathrm{Jy}$ & $12.8 \mathrm{Jy}$ & $12.7 \mathrm{Jy}$ & $12.7 \mathrm{Jy}$ & $12.8 \mathrm{Jy}$ & $12.6 \mathrm{Jy}$ & $11.9 \mathrm{Jy}$ & $9.1 \mathrm{Jy}$ \\
\hline $\mathrm{S} \mathrm{CrA}$ & $34.1(2.5)$ & $26.7(2.7)$ & $34.2(2.9)$ & $44.2(3.9)$ & $44.7(4.3)$ & $52.4(4.7)$ & $50.5(7.5)$ & $39.7(6.6)$ \\
\hline & $16.9 \mathrm{Jy}$ & $16.1 \mathrm{Jy}$ & $15.7 \mathrm{Jy}$ & $15.3 \mathrm{Jy}$ & $15.0 \mathrm{Jy}$ & $14.7 \mathrm{Jy}$ & $13.4 \mathrm{Jy}$ & $9.8 \mathrm{Jy}$ \\
\hline DG Tau & $46.4(2.0)$ & $42.5(2.0)$ & $45.9(2.3)$ & $50.0(2.9)$ & 47.7 (2.9) & $44.9(3.5)$ & $67.7(4.5)$ & $31.0(3.9)$ \\
\hline & $17.6 \mathrm{Jy}$ & $16.9 \mathrm{Jy}$ & $16.8 \mathrm{Jy}$ & $16.5 \mathrm{Jy}$ & $16.2 \mathrm{Jy}$ & $15.9 \mathrm{Jy}$ & $14.4 \mathrm{Jy}$ & $10.4 \mathrm{Jy}$ \\
\hline RU Lup & $<9.0$ & $10.8(2.9)$ & $11.5(2.1)$ & - & - & - & - & - \\
\hline & $4.2 \mathrm{Jy}$ & $4.0 \mathrm{Jy}$ & $4.1 \mathrm{Jy}$ & - & - & - & - & - \\
\hline
\end{tabular}

Notes. ${ }^{(a)}$ The transition $J=31 \rightarrow 30$ is a blend with $\mathrm{OH}$, so the values listed are upper limits. The transitions $J=26 \rightarrow 25$ at $100.46 \mu \mathrm{m}$ and $J=$ $13 \rightarrow 12$ at $200.41 \mu \mathrm{m}$, are not listed, as the data are too noisy to give a meaningful upper limit.

Paper I). Furthermore, sources with higher [O I] 63 fluxes also have higher far-IR CO fluxes, as is shown in Meeus et al. (2012), for the $\mathrm{CO} J=18 \rightarrow 17$ transition.

In HAEBEs, the bulk of the UV radiation is stellar, and the relative contribution from accretion is small. In contrast, the accretion luminosity is the most important contributor to the UV luminosity of TTS (e.g., Yang et al. 2012), as the relatively cool stellar photospheres produce very little UV flux. In Sect. 3, we showed that $T_{\text {rot }}$ is higher in our sample of TTS than in the HAEBEs. This can perhaps be attributed to a difference in the gas heating mechanism, which is stellar in HAEBEs, while it is due to accretion and possibly to shocks in the TTS. Podio et al. (2012) and Karska et al. (2013) show that heating by shocks is necessary to explain the observed molecular line emission 

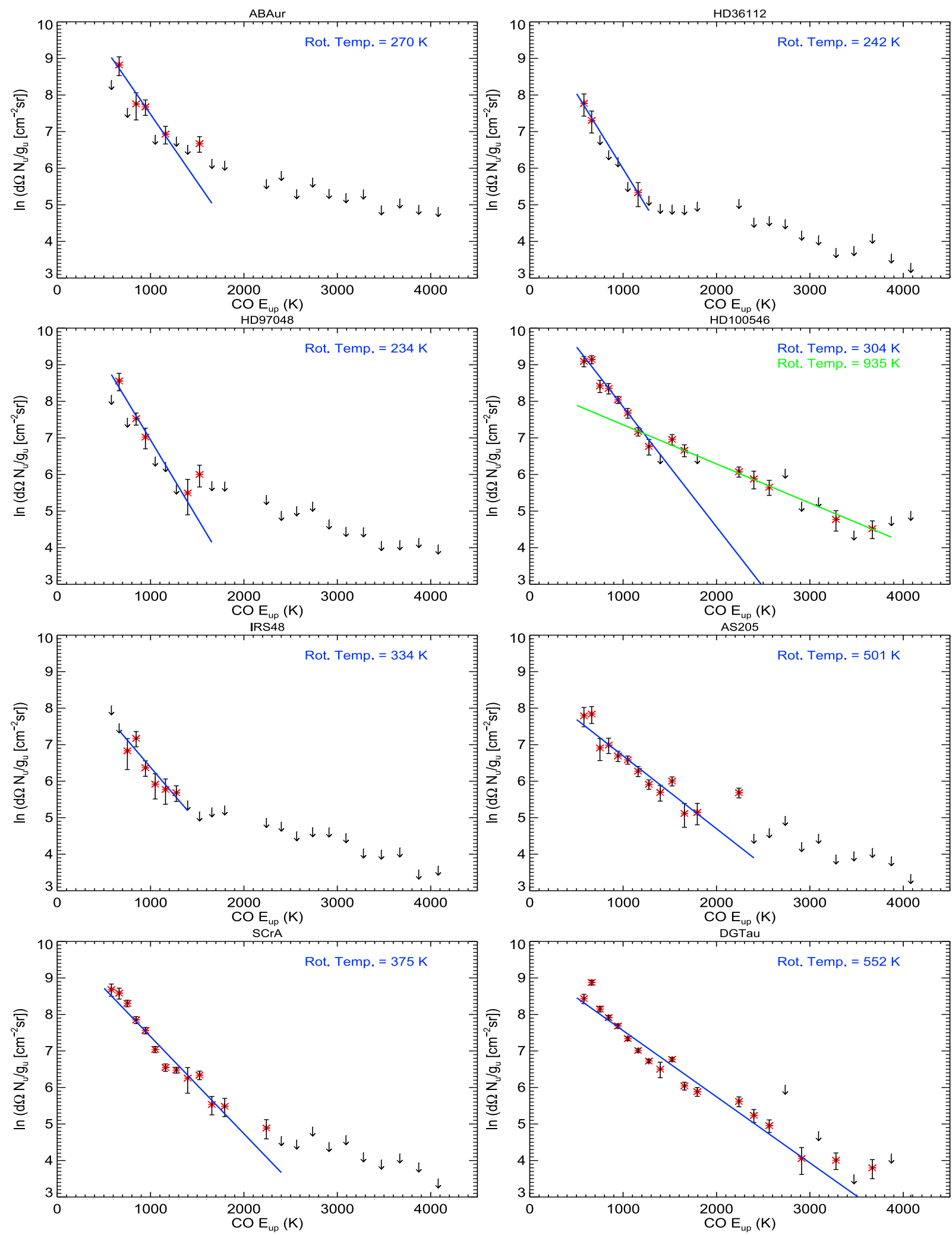

Fig. 2. Rotational diagrams of the sources with at least $3 \mathrm{CO}$ emission line detections (marked with a red asterisk, upper limits with an arrow). We also show a fit to the ladder, from which the rotational temperature can be derived.

in outflow sources. In our TTS sample, at least two sources with CO detections (DG Tau and RU Lup) show evidence of a jet.

Given the differences in origin of the UV radiation, which are important in the context of gas heating mechanisms, we discuss HAEBEs and TTS separately in the following sections.

\subsubsection{HAEBEs}

The five HAEBEs with CO detections all have flaring discs with a hot disc atmosphere. Four out of five detections are in stars with a relatively high $L_{\mathrm{UV}}$, but there are also non-detections at $L_{\mathrm{UV}}$ values that are similar to those of the detections, such as 
Table 3. $T_{\text {rot }}$ derived from the rotational diagrams.

\begin{tabular}{lll}
\hline \hline Object & $T_{\text {rot }}(\mathrm{K})$ & Notes \\
\hline AB Aur & $270_{-39}^{+53}$ & Highest $E_{\text {up }}$ not fitted, 2 components? \\
HD 36112 & $242_{-31}^{+41}$ & \\
HD 97048 & $234_{-27}^{+36}$ & Highest $E_{\text {up }}$ not fitted, 2 components? \\
HD 100546 & $276_{-17}^{+19}$ & Needs 2 temperature components \\
& $935_{-69}^{+81}$ & \\
IRS 48 & $334_{-50}^{+71}$ & \\
\hline AS 205 & $501_{-36}^{+41}$ & Highest $E_{\text {up }}$ not fitted, 2 components? \\
S CrA & $375_{-12}^{+14}$ & \\
DG Tau & $582_{-11}^{+12}$ & \\
\hline
\end{tabular}

Table 4. Parameters employed in the thermo-chemical models

\begin{tabular}{ll}
\hline \hline Parameter & Value \\
\hline Disc mass & $M_{\text {disc }}=0.1,10^{-2}, 10^{-3}, 10^{-4} M_{\odot}$ \\
Flaring angle & $\psi=0.0,0.1,0.2$ \\
Inner radius & $r_{\text {in }}=0.383 \mathrm{AU}^{a}$ \\
Outer radius & $r_{\text {out }}=400 \mathrm{AU}$ \\
Scale height at 100 AU & $H_{100 \mathrm{AU}}=10 \mathrm{AU}$ \\
Surface density power-law & $q=-1$ \\
Stellar luminosity & $L_{*}=30 L_{\odot}$ \\
Stellar temperature & $T_{*}=10^{4} \mathrm{~K}$ \\
Stellar X-ray luminosity & $L_{\mathrm{X}}=10^{29} \mathrm{erg} \mathrm{s}^{-1}$ \\
Gas-to-dust mass ratio & $g / d=100$ \\
Distance & $d=150 \mathrm{pc}$ \\
Inclination & $i=30^{\circ}$ \\
Grain size & $a=0.1-10 \mu \mathrm{m}$ \\
Grain size distribution & $\mathrm{d} n / \mathrm{d} a \propto a^{-3.5}$ \\
Grain composition & $\mathrm{ISM}($ Weingartner \& Draine 2001) \\
\hline
\end{tabular}

Notes. ${ }^{(a)}$ Dust sublimation radius, $0.07 \sqrt{L_{*} / L_{\odot}} \mathrm{AU}$ (Dullemond et al. 2001).

the transitional disc HD 141569 A. Unfortunately, the modest ranges in $L_{\mathrm{UV}}$ and line luminosity coupled with the small sample size make it impossible to statistically test whether the line luminosity correlates with $L_{\mathrm{UV}}$.

\subsubsection{TTS}

Salyk et al. (2013) show that the accretion rate can also be correlated with the strength of the $\operatorname{HPf} \beta(n=7 \rightarrow 4)$ line at $4.65 \mu \mathrm{m}$, which was observed in all of the TTS in our sample. In the following, we use data from that paper, taken with either VLT/CRIRES (Pontoppidan et al. 2011) or Keck/NIRSPEC. In Fig. 3, we plot the $\operatorname{HPf} \beta$ line emission both for sources that do show the PACS CO lines and those that do not. All the sources with $\mathrm{CO}$ detections have strong $\operatorname{H} \operatorname{Pf} \beta$ lines, while most of the sources without $\mathrm{CO}$, except for RNO 90, have weak $\mathrm{H} \operatorname{Pf} \beta$ lines. The ro-vibrational $\mathrm{CO}$ lines also appear to be stronger in the sources with PACS detections, especially for the $v=2 \rightarrow 1 \mathrm{R}(8)$ line at $4.6598 \mu \mathrm{m}$, which is enhanced by UV radiative excitation (Brown et al. 2013). This implies a connection between accretion rate and $\mathrm{CO}$ detections. This is perhaps due to the UV radiation provided by the accretion column. However, a direct connection with the value of $L_{\mathrm{acc}}$ is not found. We see a similar spread in $L_{\text {acc }}$ for the detections and the non-detections, so that there is no clear correlation between these variables.

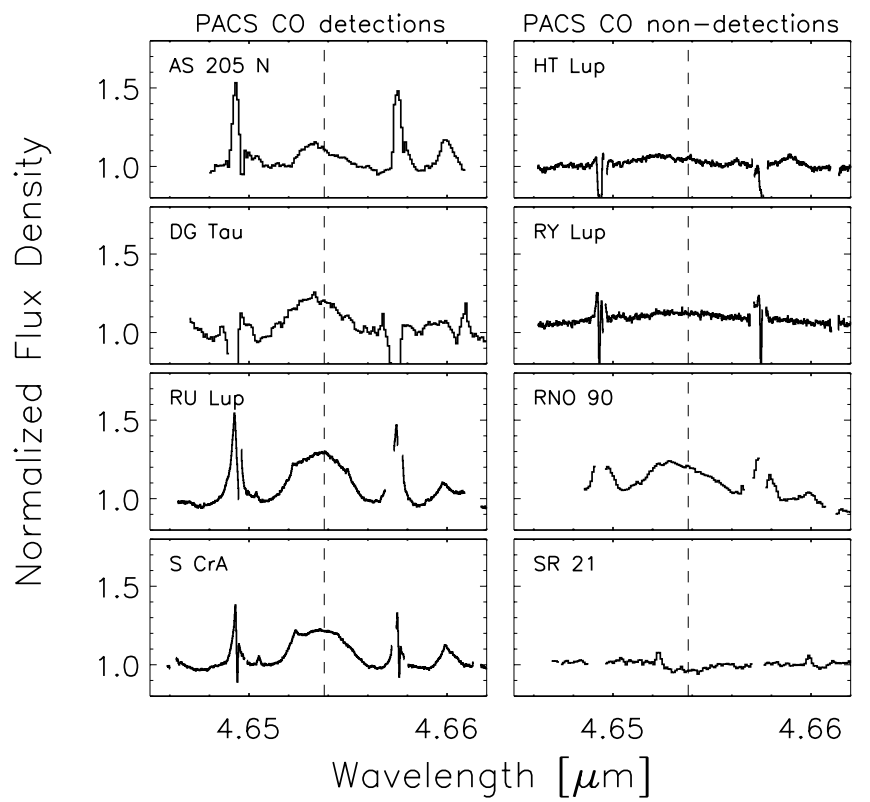

Fig. 3. Observations of $\operatorname{HPf} \beta$ for the TTS sample; the line position is indicated with the vertical dashed line. Left: sources for which we have $\mathrm{CO}$ detections; right: sources for which $\mathrm{CO}$ was not detected with PACS.

\subsection{Thermo-chemical models of the mid- to high-J cO emission.}

To explain the trend that far-IR CO lines are only detected towards flared discs, we ran a grid of thermo-chemical models introduced by Bruderer et al. (2012). These models solve for the dust radiative transfer, chemistry, and thermal balance in a selfconsistent way. Details of the model and benchmark tests are reported in BR12. In that paper, the mid- to high- $J \mathrm{CO}$ emission of HD 100546 is reproduced with the fine structure lines of [O I] for a model tailored specifically to that disc. Here, we run a grid of generic disc models using a parameterised density structure. The surface density profile $\Sigma(r)$ of the disc varies as

$\Sigma(r) \propto r^{q}$

while the vertical density structure $\rho(r, z)$ is a Gaussian

$\rho(r, z) \propto \Sigma(r) \cdot \exp \left(-1 / 2(z / H(r))^{2}\right)$.

The scale-height $H(r)$ is given by the power-law

$H(r)=H_{0}\left(\frac{r}{100 \mathrm{AU}}\right)^{\psi+1}$.

The parameters assumed in the grid are given in Table 4 . We vary the disc mass $M_{\text {disc }}$ and the flaring parameter $\psi$ to study the dependence of the $\mathrm{CO}$ lines on these two parameters.

As a word of caution, we note that thermo-chemical modelling is prone to large uncertainties, particularly in the gas temperature calculation (Röllig et al. 2007). We thus refrain from fitting individual objects but aim to reproduce the main trends found in the observations.

How does the flaring parameter affect the temperature structure? In Fig. 4, the density, gas-temperature, and $\mathrm{CO}$ abundance profile of models with varying flaring parameters. $\psi=0.0,0.1$ and 0.2 , and $M_{\text {disc }}=10^{-2} M_{\odot}$, are shown. The figures are plotted such that the axes are the distance-normalised height $(z / r)$ versus $\log (r)$. In the model without flaring $(\psi=0.0)$, there is always an attenuating column of gas and dust between the star and regions 

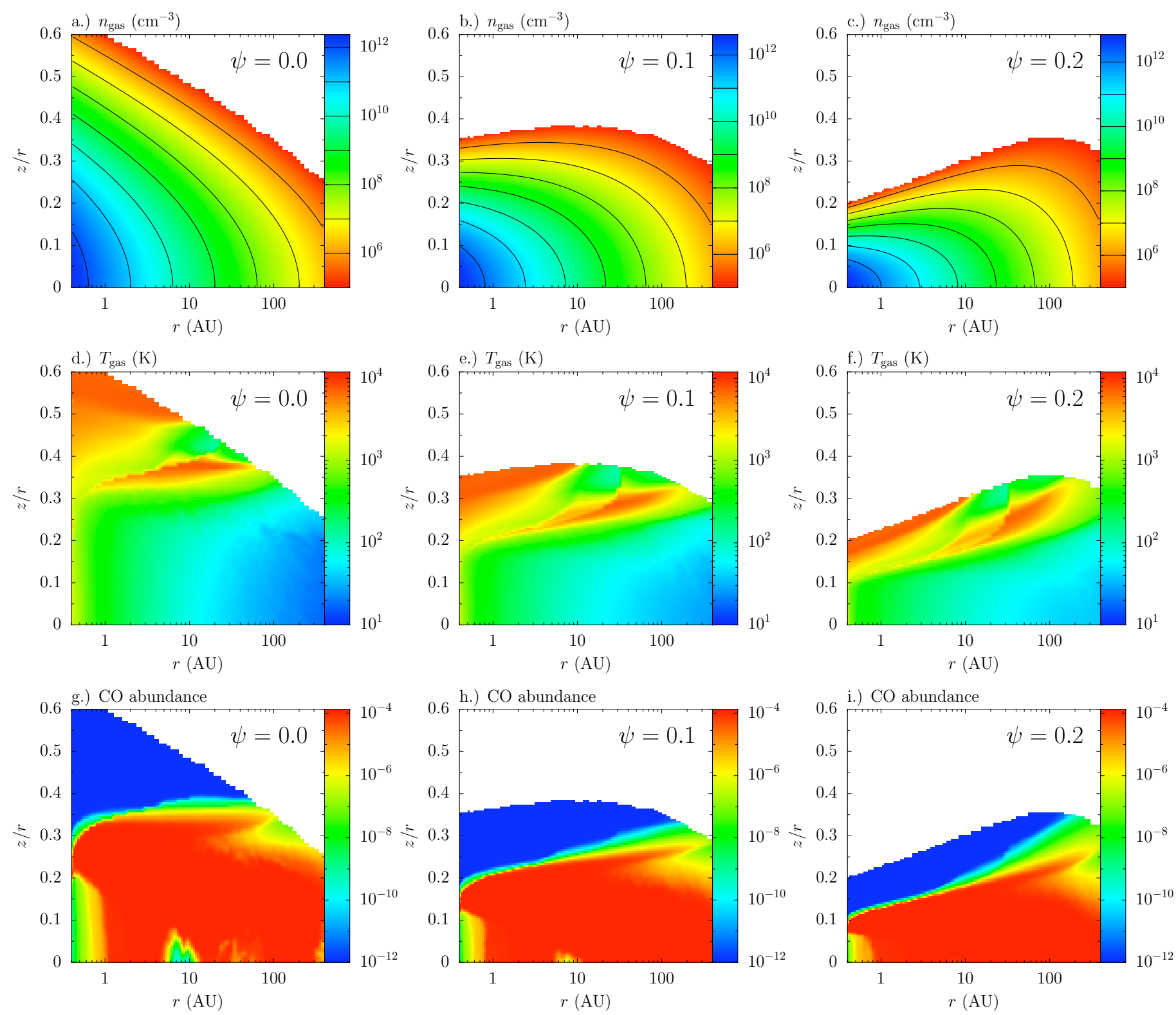

Fig. 4. Model gas density ( $n_{\text {gas }}$, top panels) gas-temperature $\left(T_{\text {gas }}\right.$, center panels $)$ and CO abundance $\left(n_{\mathrm{CO}} / n_{\text {gas }}\right.$, bottom panels) of thermo-chemical models with $M_{\text {disc }}=10^{-2} M_{\odot}$ and different flaring parameter $\psi=0.0,0.1$ or 0.2 (left to right columns).

with density $\gtrsim 10^{6} \mathrm{~cm}^{-3}$. This density corresponds to the critical density of the CO $J=16-15$ transition $\left(E_{\text {up }}=750 \mathrm{~K}\right)$, which is needed to excite the line collisionally. The mass of gas, which has a temperature high enough $(T>750 \mathrm{~K})$ to excite the PACS $\mathrm{CO}$ lines, $M_{\mathrm{CO} \text { ex }}$, is always small compared to the total disc mass $(<0.5 \%)$. However, more gas can be irradiated and heated directly by the star to temperatures high enough for the excitation of the high- $J \mathrm{CO}$ lines for larger flaring parameters. For $\psi=0.0$, $M_{\mathrm{COex}}=4.2 \times 10^{-6} M_{\odot} ;$ for $\psi=0.1, M_{\mathrm{COex}}=2.0 \times 10^{-5} M_{\odot}$; and for $\psi=0.2, M_{\mathrm{COex}}=4.5 \times 10^{-5} M_{\odot}$ : an increase up to a factor 10 with increasing flaring parameter $\psi$. Furthermore, the radial extent of the warm region also increases with amount of flaring. This is of importance, since lines up to $J=16-15$ can be optically thick so that the size of the emitting area determines the amount of line flux (see BR12).

The abundance of CO (Figs. 4g, h, and i) is dominated by photodissociation in the upper atmosphere and the inner rim of the disc. The "warm-finger" of $\mathrm{CO}$ is produced by $\mathrm{CO}$ formation through the reaction of $\mathrm{C}^{+}$with $\mathrm{H}_{2}$ to $\mathrm{CH}^{+}$, which only proceeds at high gas temperature (Jonkheid et al. 2007; Agúndez et al. 2008). Starting from $\mathrm{CH}^{+}$, a chain of reactions through $\mathrm{CO}^{+}$ and $\mathrm{HCO}^{+}$then leads to $\mathrm{CO}$. The species $\mathrm{CH}^{+}$is detected in both HD 97048 and HD 100546 (Thi et al. 2011; Paper I). The CO lines discussed in this section form at large heights in the disc atmosphere and are not affected by any chemistry lower in the disc.

The CO ladder derived from the grid of models is shown in Fig. 5. Lines with $J_{\text {up }} \leq 7$ are convolved to the beam of the APEX telescope, which has been used to detect these lines towards Herbig discs (e.g., Panić et al. 2010). Higher $J_{\text {up }}$ lines are convolved to the beam of the PACS instrument. Typical PACS detection limits are shown by the example of AB Aur. We find that the flux in the PACS lines indeed depends considerably on the flaring of the disc. This is a direct result of the increasing mass of warm gas at several $100 \mathrm{~K}$ with the flaring parameter $\psi$.

On the other hand, the shape of the CO ladder does not vary significantly with disc mass, as long as $M_{\text {disc }} \geq 10^{-3} M_{\odot}$. While the CO lines above $J_{\text {up }} \sim 16$ are optically thin, the equilibrium of 

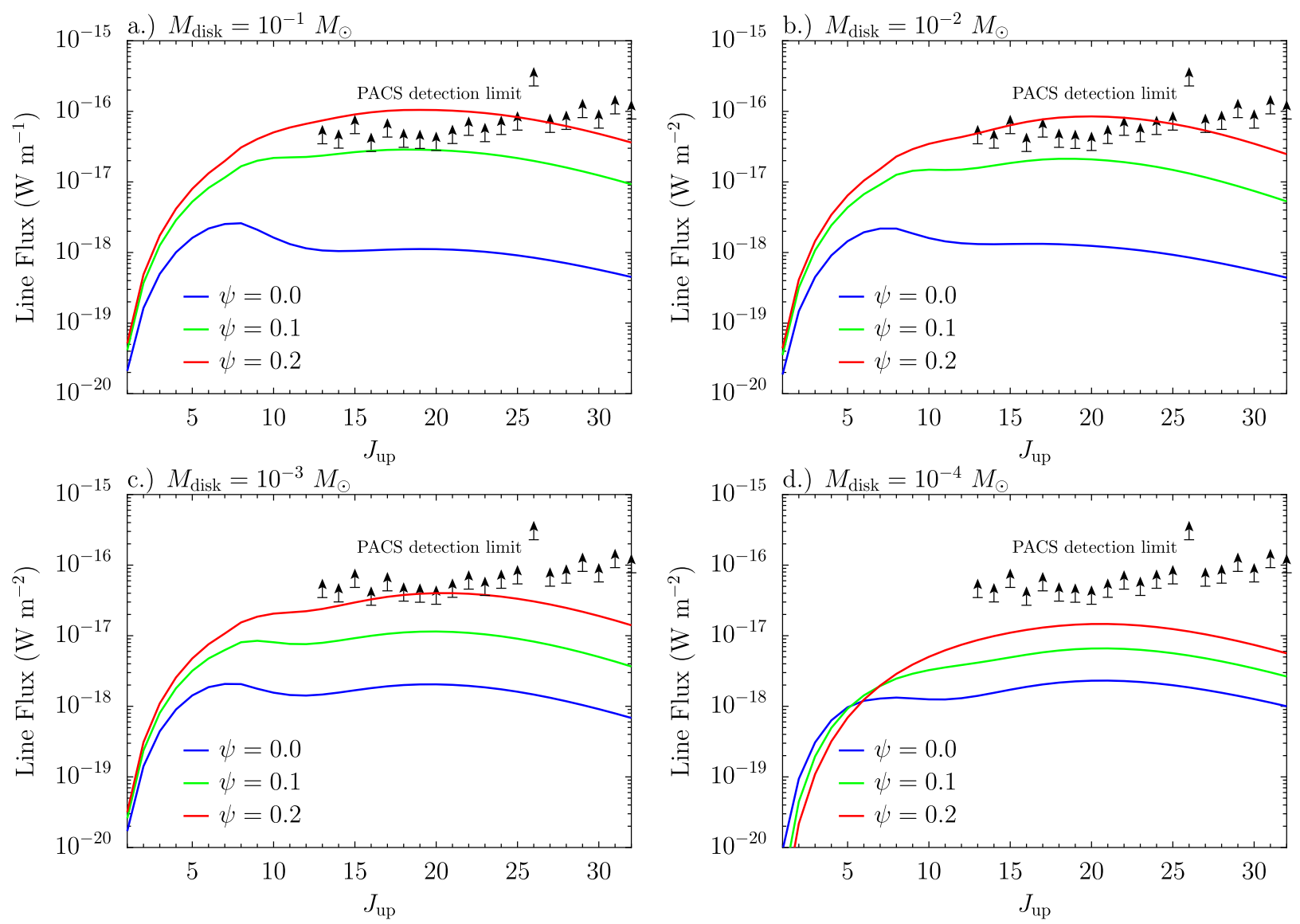

Fig. 5. Model CO ladder for the models with different gas mass $M_{\text {disc }}$ and flaring parameter $\psi$. The lines with $J_{\text {up }} \leq 7$ are convolved to the APEX beam at the appropriate frequency; the higher $J_{\text {up }}$ are convolved to PACS. Black arrows indicate the typical PACS detection limit, extracted from the AB Aur spectrum.

gas heating and cooling processes yields similar amounts of gas warm enough to excite the $\mathrm{CO}$ lines. This result is different from the findings of BR12, because they leave the dust structure unchanged and vary the gas-to-dust ratio. In the models presented here, we vary both gas and dust mass and keep the gas-to-dust ratio constant. It is the amount of dust which absorbs the impinging stellar light that is key for the heating of the gas.

From this grid of thermo-chemical models we conclude that the structure of the disc, and in particular the flaring, which allows direct stellar irradiation of a larger volume of gas, is key for the understanding of the CO ladder observed by PACS. Nonflared discs produce line emission flux that is too low to be detected in our spectra.

\section{Discussion}

What other parameters can influence the strength of the $\mathrm{CO}$ emission? In the following, we speculate about the influence of the PAH luminosity and the inner disc clearing on the far-IR $\mathrm{CO}$ emission. While PAHs are frequently detected in HAEBEs (up to 70\%; Acke et al. 2010), they are seen in up to $15 \%$ of T Tauri discs (Geers et al 2006; Oliveira et al. 2007, 2010); therefore, we concentrate our discussion on PAHs observed in HAEBEs.

\subsection{PAH luminosity}

When present, PAHs contribute significantly to disc heating, absorbing UV photons in the upper disc layer. Afterward, they heat the disc through the photoelectric effect; as such, they also have an influence on the observed line strengths (e.g., Kamp \& Dullemond 2004; Bruderer et al. 2012). Therefore, we searched for a link between the PAH luminosity and the observed $\mathrm{CO}$ emission. If we consider the sources with the highest $L_{\mathrm{UV}}\left(>4 L_{\odot}\right.$; AB Aur, HD 97048, HD 100546 - group I, and HD 141569 A, HD 150193 and HD 163296 - group II), we see that it contains equal amounts of sources with flaring and non-flaring discs, but the group II sources lack CO far-IR emission. As the amount of $L_{\mathrm{UV}}$ does not solely determine the strength of the CO lines, we now also consider the PAH emission. In Table 1 , we list $L_{\mathrm{PAH}}$ for those objects where PAH bands are detected, while we plot the $L_{\mathrm{PAH}}$ as a function of $L_{\mathrm{UV}}$ for both sources with and without CO detections in Fig. 6. The group II sources HD 150193 and HD 163296 do not show PAH emission features, while the transitional disc HD 141569 A only has weak PAH bands when compared to the group I sources: $L_{\mathrm{PAH}} \sim 0.007 L_{\odot}$ versus 0.030 to $0.39 L_{\odot}$ for the 5 HAEBEs with $\mathrm{CO}$ detections. This suggests that strong PAH emission, in addition to strong UV luminosity, enhances CO far-IR emission, which is consistent with the non-detection in HD 141569 A. However, the PAH luminosity does not uniquely determine the $\mathrm{CO}$ line flux. It is interesting in this context to mention studies of the ro-vibrational bands of $\mathrm{CO}$ at $4.7 \mu \mathrm{m}$, revealing that HD 97048, HD 100546, and HD 141569 A have UV fluorescence, so that $T_{\text {vib }}>5600 \mathrm{~K}$, while their $T_{\text {rot }}$ is distinct: it is $\sim 1000 \mathrm{~K}$ for the first two and $\sim 250 \mathrm{~K}$ for HD $141569 \mathrm{~A}$ (van der Plas 2010; Brittain et al. 2007), again indicating that the PAH luminosity is an important factor in the context of gas 


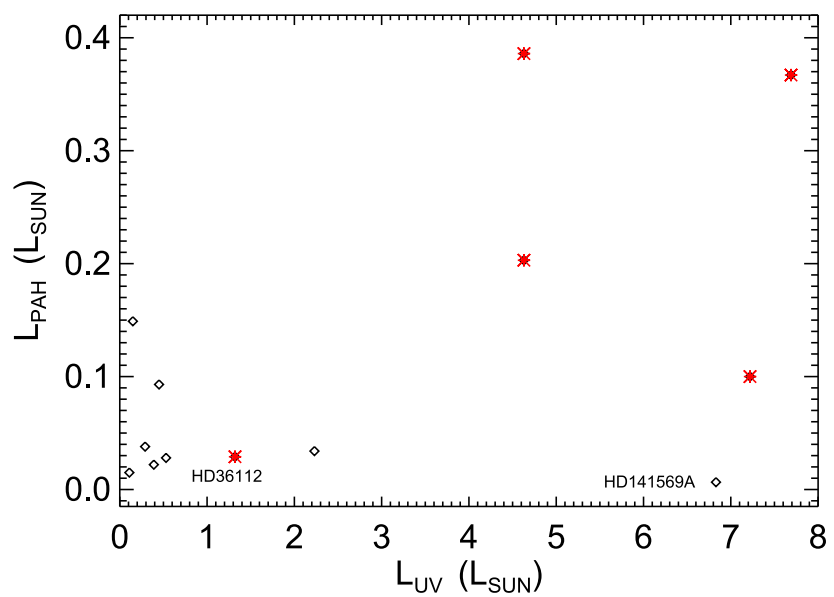

Fig. 6. $L_{\mathrm{PAH}}$ as a function of $L_{\mathrm{UV}}$ for the HAEBE sample. The red asterisks indicate sources where $\mathrm{CO}$ was detected in our spectra; the black diamonds are the sources with non-detections.

heating. However, BR12 showed that a change in the PAH abundance in their model of HD 100546 changes the high- $J$ flux by less than a dex, leaving flaring as the most important parameter determining $\mathrm{CO}$ line fluxes.

\subsection{Inner-disc clearing}

Based on a small sample of group I sources with spatially resolved mid-IR images, Maaskant et al. (2013) suggest that group I sources have dust-depleted regions in the inner disc $(\lesssim 50 \mathrm{AU})$ that let the radiation penetrate further out in the disc. As a result, the outer regions, most notably the inner edge of the outer disc, experience more heating causing an excess at midto far-IR wavelengths. On the other hand, no flat (group II) discs have reported gaps in the literature. This suggests that these discs are relatively more self-shadowed and consequently cooler in the outer parts of the disc. In our HAEBE sample with CO detections, HD 97048, HD 100546, IRS 48, HD 36112 and AB Aur all have dust-depleted inner regions (Maaskant et al. 2013 for HD 97048; Bouwman et. al. 2003 for HD 100546; Geers et al. 2007; Brown et al. 2012; and van der Marel 2013 for IRS 48; Isella et al. 2010 for HD 36112; Honda et al. 2010 for AB Aur). This indeed suggests that dust clearing in the inner disc can result in more efficient heating of the outer layers, so that the amount of warm $\mathrm{CO}$ is increased.

\subsection{Summary}

Besides dust and gas temperature decoupling in the surface layers (due to flaring) and high UV luminosity, the discussion above suggests that the CO line luminosity in HAEBEs can also be enhanced by a combination of 1) PAH heating and 2) enhanced UV penetration of the outer disc due to a dust-depleted inner region. We expect these factors to also be inter-linked, but a detailed parameter study of disc models would be needed to explain the causality.

\section{Conclusions}

We analysed PACS spectra covering 53.5 to $190 \mu \mathrm{m}$ to study the far-IR CO ladder in a sample of HAEBE and TTS. Our results can be summarised as follows:

1. Out of 22 HAEBEs and 8 TTS, we detected CO emission lines in only 5 HAEBEs (23\%) and 4 TTS (50\%).
2. In HAEBEs, the far-IR CO lines are only detected in flaring discs, indicating that the disc geometry (presence of a hot disc atmosphere) is important in creating detectable line emission. TTS with detections have evidence of a disc wind and/or outflow.

3. The highest $J$ transition $\left(J=36 \rightarrow 35\right.$ with $\left.E_{\text {up }}=3669 \mathrm{~K}\right)$ was observed in HD 100546 and the outflow source, DG Tau.

4. We constructed rotational diagrams for the objects with at least $3 \mathrm{CO}$ detections and derived $T_{\text {rot }}$ between 200 and $500 \mathrm{~K}$. The mean values of the warm components are $\left\langle T_{\text {rot }}\right\rangle=$ $271 \pm 39 \mathrm{~K}$ for the HAEBEs and $\left\langle T_{\text {rot }}\right\rangle=486 \pm 104 \mathrm{~K}$ for the TTS. The HAEBE HD 100546 is the only object that clearly needs a second, hot component of $\sim 900 \mathrm{~K}$, but other objects might also reveal a hot component when observed with a more sensitive instrument.

5. We analysed the observed line fluxes with a small grid of thermo-chemical models. We found that an increased amount of flaring increased the CO line flux; a certain amount of flaring is necessary for the lines to become detectable with PACS and can increase the line flux by a factor 10. This is a direct result of the increase in warm gas mass with increasing amount of flaring when $T_{\text {gas }}$ and $T_{\text {dust }}$ are decoupled. Furthermore, we found that the disc mass is not a sensitive parameter, as long as it is higher than a few $10^{-4} M_{\odot}$.

6. For the HAEBEs amongst the group I sources, the ones with highest $L_{\mathrm{UV}}$ have detectable $\mathrm{CO}$ lines.

7. In TTS, sources with strong $\operatorname{HPf} \beta$ lines (a tracer of accretion rate) tend to have $\mathrm{CO}$ emission possibly because of the UV heating provided by the accretion column. However, a quantitative connection between $L_{\mathrm{acc}}$ and CO detections is not yet clear.

8. We suggest that both strong PAH luminosity and the presence of a dust-depleted inner disc region can increase disc heating and by consequence, enhance the far-IR CO line luminosities.

Acknowledgements. We would like to thank the PACS instrument team for their dedicated support. G. Meeus is supported by Ramon y Cajal grant RYC-201107920. B. Montesinos is partly supported by AYA-2011-26202. Support for this work, part of the Herschel "Open Time Key Project" Program, was provided by NASA through an award issued by the Jet Propulsion Laboratory, California Institute of Technology. PACS has been developed by a consortium of institutes led by MPE (Germany) and including UVIE (Austria); KUL, CSL, IMEC (Belgium); CEA, OAMP (France); MPIA (Germany); IFSI, OAP/AOT, OAA/CAISMI, LENS, SISSA (Italy); IAC (Spain). This development has been supported by the funding agencies BMVIT (Austria), ESA-PRODEX (Belgium), CEA/CNES (France), DLR (Germany), ASI (Italy), and CICT/MCT (Spain). This paper is based (in part) on observations made with ESO Telescopes at the Paranal Observatory under program ID 179.C-0151. This research has made use of the SIMBAD database, operated at CDS, Strasbourg, France.

\section{References}

Acke, B., Bouwman, J., Juhász, A., et al. 2010, ApJ, 718, 558 Agúndez, M., Cernicharo, J., \& Goicoechea, J. R. 2008, A\&A, 483, 831 Bary, J. S., Weintraub, D. A., Shukla, S. J., et al. 2008, ApJ, 678, 1088 Bast, J. E., Brown, J. M., Herczeg G. J., et al. 2011, A\&A, 527, A119 Benisty, M., Tatulli, E., Ménard, F., \& Swain, M. R. 2010, A\&A, 511, A75 Bitner, M. A., Richte, M. J., Lacy, J. H., et al. 2008, ApJ, 688, 1326 Blake, G. A., \& Boogert, A. C. A. 2004, ApJ, 606, 73

Bouwman, J., de Koter, A., Dominik, C., \& Waters, L. B. F. M. 2003, A\&A, 401, 577

Brittain, S. D., Simon, Th. , Najita, J. R., \& Rettig, T. W. 2007, ApJ, 659, 685 Brown, J. M., Herczeg, G. J., Pontoppidan, K. M., \& van Dishoeck E. F. 2012, ApJ, 744, 116

Brown, J. M., Pontoppidan, K. M., van Dishoeck, E. F., et al. 2013, ApJ, 770, 94 
Bruderer, S., van Dishoeck, E. F., Doty, S. D., \& Herczeg, G. J. 2012, A\&A, 541, A91

Carmona, A., van den Ancker, M. E., Henning, Th., et al. 2008, A\&A, 477, 839

Carmona, A., van der Plas, G., van den Ancker, M. E., et al. 2011, A\&A, 533, A39

Dent, W. R. F., Greaves, J. S., \& Coulson, I. M. 2005, MNRAS, 359, 663

Dominik, C., Dullemond, C. P., Waters, L. B. F. M., et al. 2003, A\&A, 398, 607 Dullemond, C. P., Dominik, C., \& Natta, A. 2001, ApJ, 560, 957

Fedele, D., Bruderer, S., van Dischoeck, E. F., et al. 2013, A\&A, 559, A77

France, K., Schindhelm, E., Herczeg, G. J., et al. 2012, ApJ, 756, 171

Geers, V. C., Augereau, J.-C., Pontoppidan, K. M., et al. 2006, A\&A, 459, 545

Geers, V. C., Pontoppidan, K. M., van Dishoeck, E. F., et al. 2007, A\&A, 476, 279

Giannini, T., Lorenzetti, D., Tommasi, E., et al. 1999, A\&A, 346, 617

Goldsmith, P. F., \& Langer, W. D. 1999, ApJ, 517, 209

Goto, M., Usuda, T., Dullemond, C. P., et al. 2006, ApJ, 652, 758

Green, J. D., Evans, N. J. II, Jörgensen, J. K., et al. 2013, ApJ, 770, 123

Herczeg, G. J., Walter, F. M., Linsky, J. L., et al. 2005, AJ, 129, 2777

Herczeg, G. J., Linsky, J. L., Jeffrey, L., et al. 2006, ApJS, 165, 256

Hersant, F., Wakelam, V., Dutrey, A., et al. 2009, A\&A, 493, L49

Honda, M., Inoue, A. K., Okamoto, Y. K., et al. 2010, ApJ, 718, 199

Ingelby, L., Calvet, N., Bergin, E., et al. 2011, ApJ, 743, 105

Isella, A., Natta, A., Wilner, D., Carpenter, J. M., \& Testi, L. 2010, ApJ, 725, 1735

Jonkheid, B., Dullemond, C. P., Hogerheijde M. R., \& van Dishoeck, E. F. 2007, A\&A, 463, 203

Juhász, A., Bouwman, J. Henning, Th., et al. 2010, ApJ, 721, 431

Kamp, I., \& Dullemond, C. P. 2004, ApJ, 615, 991

Kamp, I., Woitke, P., Pinte, C., et al. 2011, A\&A, 532, A85

Karska, A., Herczeg, G. J., van Dishoeck, E. F., et al. 2013, A\&A, 552, A141

Koerner, D. W., \& Sargent, A. I. 1995, AJ, 109, 2138

Lahuis, F., van Dishoeck, E. F., Blake, G. A., et al. 2007, ApJ, 665, 492

Lavalley-Fouquet, C., Cabrit, S., \& Dougados, C. 2000, A\&A, 356, L41

Maaskant, K. M., Honda, M., Waters, L. B. F. M., et al. 2013, A\&A, 555, A64
Martin-Zaïdi, C., Habart, E., Augereau, J.-C., et al. 2009, ApJ, 695, 1302 Martin-Zaïdi, C., Augereau, J.-C., Ménard, F., et al. 2010, A\&A, 516, A110 Meeus, G., Waters, L. B. F. M., Bouwman, J., et al. 2001, A\&A, 365, A476 Meeus, G., Montesinos, B., Mendigutía, I., et al. 2012, A\&A, 544, A78 Mundt, R. 1984, ApJ, 280, 749

Najita, J., Carr, J. S., \& Mathieu, R. D. 2003, ApJ, 589, 931

Öberg, K. I., Qi, C., Fogel, J. K. J., et al. 2010, ApJ, 720, 480 Panić, O., \& Hogerheijde 2009, A\&A, 508, 707

Panić, O., Hogerheijde, M. R., Wilner, D., \& Qi, C. 2008, A\&A, 491, 219

Panić, O., van Dishoeck, E. F., Hogerheijde, M. R., et al. 2010, A\&A, 519, A110

Pilbratt, G. L., Riedinger, J. R., Passvogel, T., et al. 2010, A\&A, 518, L1

Podio, L., Kamp, I., Flower, D., et al. 2012, A\&A, 545, A44

Poglitsch, A., Waelkens, C., Geis, N., et al. 2010, A\&A, 518, L2

Pontoppidan, K. M., Blake, G. A., van Dishoeck, E. F., et al. 2008, ApJ, 684, 1323

Pontoppidan, K. M., van Dishoeck, E. F., Blake, G. A., et al. 2011, The Messenger, 143, 32

Röllig, M., Abel, N. P., Bell, T., et al. 2007, A\&A, 467, 187

Salyk, C., Blake, G. A., Boogert, A. C. A., \& Brown, J. M. 2009, ApJ, 699, 330

Salyk, C., Blake, G. A., Boogert, A. C. A., \& Brown, J. M. 2011, ApJ, 743, 112

Salyk, C., Herczeg, G. J., Brown, J. M., et al. 2013, ApJ, 769, 21

Sturm, B., Bouwman, J., Henning, T., et al. 2010, A\&A, 518, L129

Thi, W. F., van Dishoeck, E. F., Blake, G. A., et al. 2001, ApJ, 561, 1074

Thi, W. F., Ménard, F., Meeus, G., et al. 2011, A\&A, 530, L2

Thi, W. F., Kamp, I., Woitke, P., et al. 2013, A\&A, 551, A49

van de Marel, N., van Dishoeck, E. F., Bruderer, S., et al. 2013, Science, 340 1199

van der Plas, G. 2010, Ph.D. Thesis (University of Amsterdam)

van der Plas, G., van den Ancker, M. E., Acke, B., et al. 2009, A\&A, 500, 1137

Weingartner, J. C., \& Draine, B. T. 2001, ApJ, 548, 296

Williams, J. P., \& Cieza, L. A. 2011, ARA\&A, 49, 67

Woitke, P., Pinte, C., Tilling, I., et al. 2010, MNRAS, 405, L26

Yang, H., Herczeg, G. J., Linsky, J. L., et al. 2012, ApJ, 744, 121 


\section{Appendix A: Detection of CO transitions}

In Table A.1, we list the transitions that were detected in our PACS spectra, while we show windows around the position of the $\mathrm{CO}$ lines for those objects where $\mathrm{CO}$ line emission was detected in Figs. A.1 to A.8.

Table A.1. CO Transitions detected in the PACS SED scans.

\begin{tabular}{ccc}
\hline \hline Transition & Wavelength $(\mu \mathrm{m})$ & $E_{\text {up }}(\mathrm{K})$ \\
\hline$J 36 \rightarrow 35$ & 72.84 & 3669 \\
$J 35 \rightarrow 34$ & 74.89 & 3471 \\
$J 34 \rightarrow 33$ & 77.11 & 3279 \\
$J 33 \rightarrow 32$ & 79.36 & 3093 \\
$J 32 \rightarrow 31$ & 81.81 & 2911 \\
$J 31 \rightarrow 30$ & 84.41 & 2735 \\
$J 30 \rightarrow 29$ & 87.19 & 2565 \\
$J 29 \rightarrow 28$ & 90.16 & 2400 \\
$J 28 \rightarrow 27$ & 93.35 & 2240 \\
$J 25 \rightarrow 24$ & 104.45 & 1794 \\
$J 24 \rightarrow 23$ & 108.76 & 1657 \\
$J 23 \rightarrow 22$ & 113.46 & 1524 \\
$J 22 \rightarrow 21$ & 118.58 & 1397 \\
$J 21 \rightarrow 20$ & 124.19 & 1276 \\
$J 20 \rightarrow 19$ & 130.37 & 1160 \\
$J 19 \rightarrow 18$ & 137.20 & 1050 \\
$J 18 \rightarrow 17$ & 144.78 & 945 \\
$J 17 \rightarrow 16$ & 153.27 & 846 \\
$J 16 \rightarrow 15$ & 162.81 & 752 \\
$J 15 \rightarrow 14$ & 173.63 & 663 \\
$J 14 \rightarrow 13$ & 186.00 & 581 \\
\hline
\end{tabular}


G. Meeus et al.: $\mathrm{CO}$ in protoplanetary discs
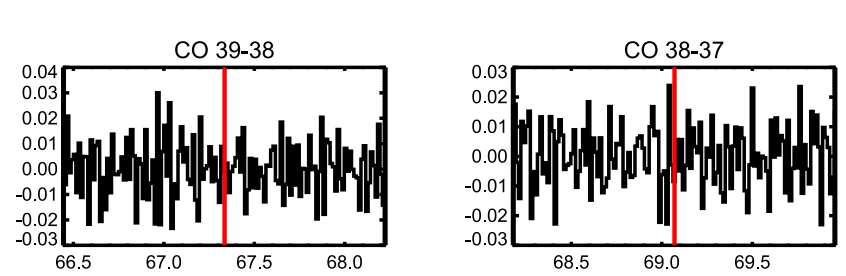

ABAur
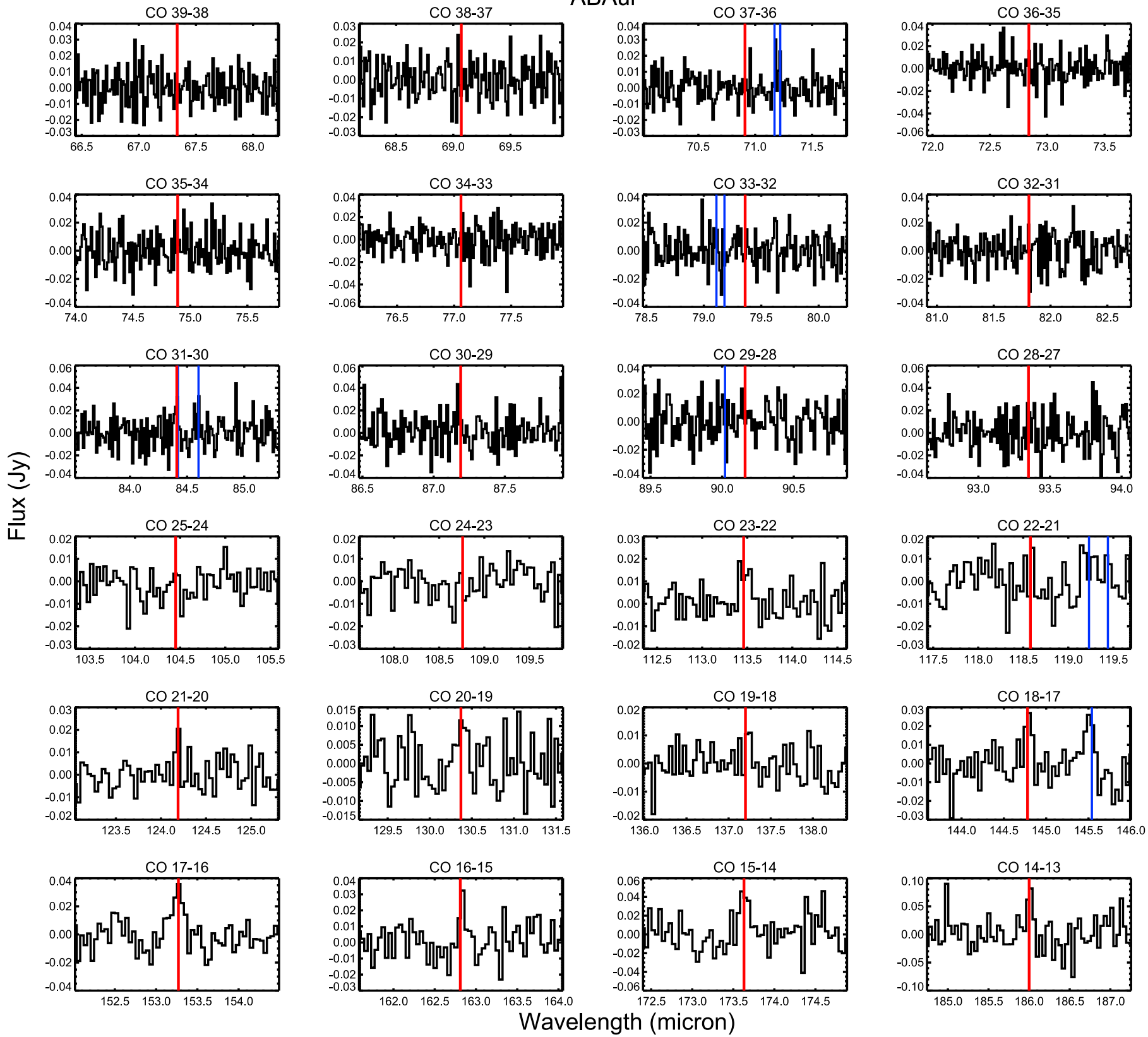

Fig. A.1. Spectra of $\mathrm{AB}$ Aur, centered on the $\mathrm{CO}$ lines, indicated in red. The $\mathrm{OH}$ line at $84.42 \mu \mathrm{m}$ blends with the $\mathrm{CO} J=31 \rightarrow 30$ line. In blue, we indicate the positions of other lines $\left(\mathrm{CH}^{+}\right.$at $90 \mu \mathrm{m}$ and [O I] at $145 \mu \mathrm{m}$; rest are $\mathrm{OH}$ doublets). 

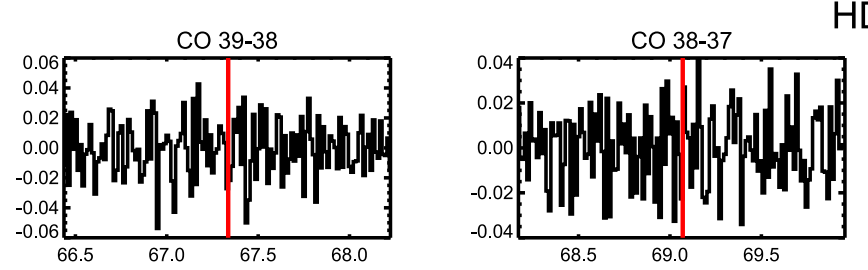

HD36112
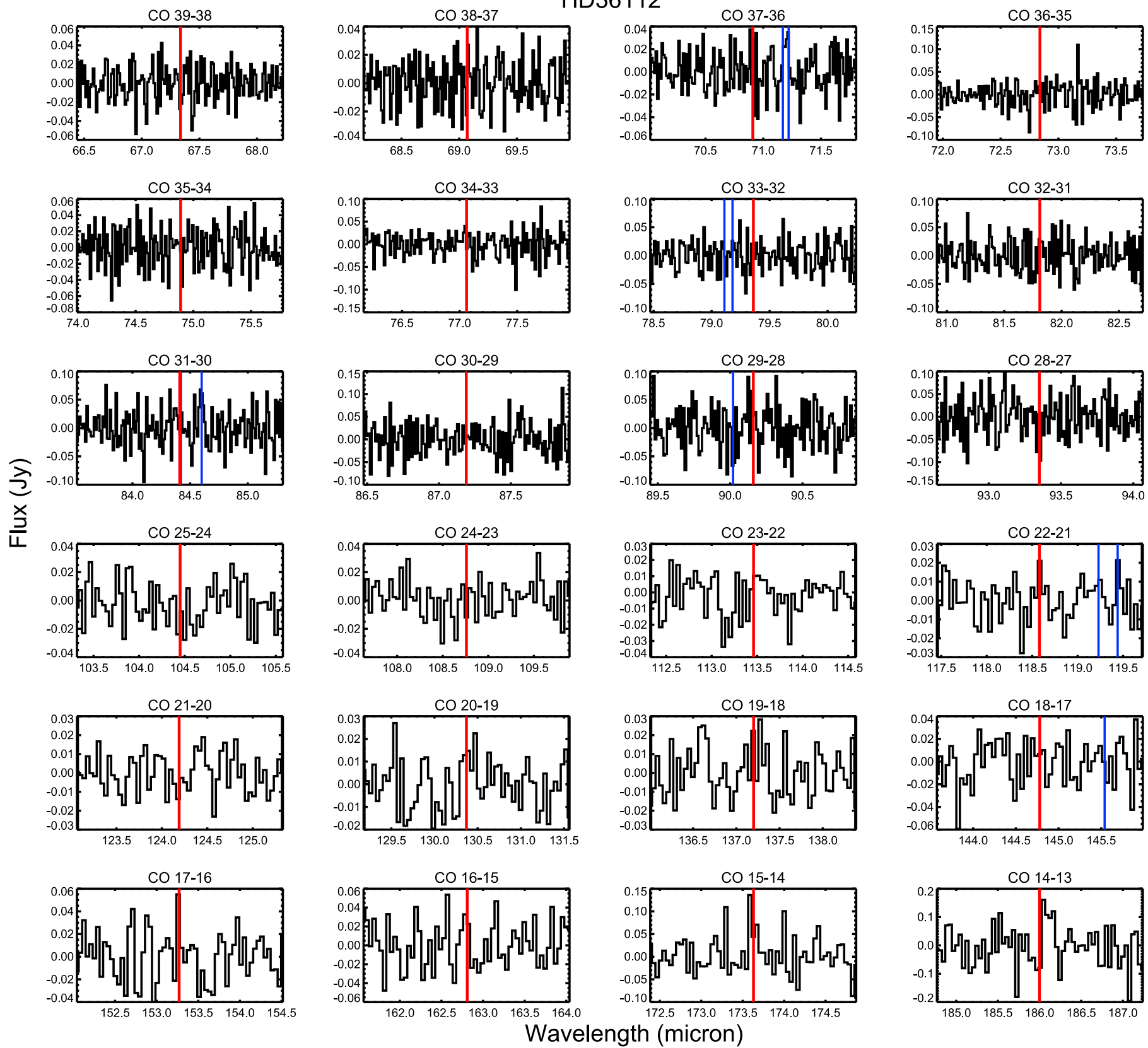

Fig. A.2. Spectra of HD 36112, centered on the $\mathrm{CO}$ lines, indicated in red. The $\mathrm{OH}$ line at $84.42 \mu \mathrm{m}$ blends with the $\mathrm{CO} J=31 \rightarrow 30$ line. In blue, we indicate the positions of other lines $\left(\mathrm{CH}^{+}\right.$at $90 \mu \mathrm{m}$ and $\left[\mathrm{O}_{\mathrm{I}}\right]$ at $145 \mu \mathrm{m}$; rest are $\mathrm{OH}$ doublets). 
G. Meeus et al.: $\mathrm{CO}$ in protoplanetary discs
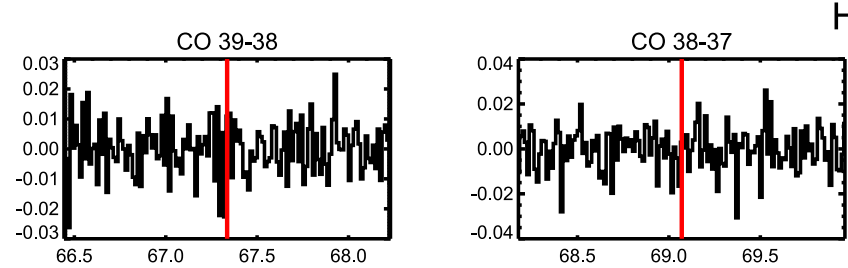

HD97048
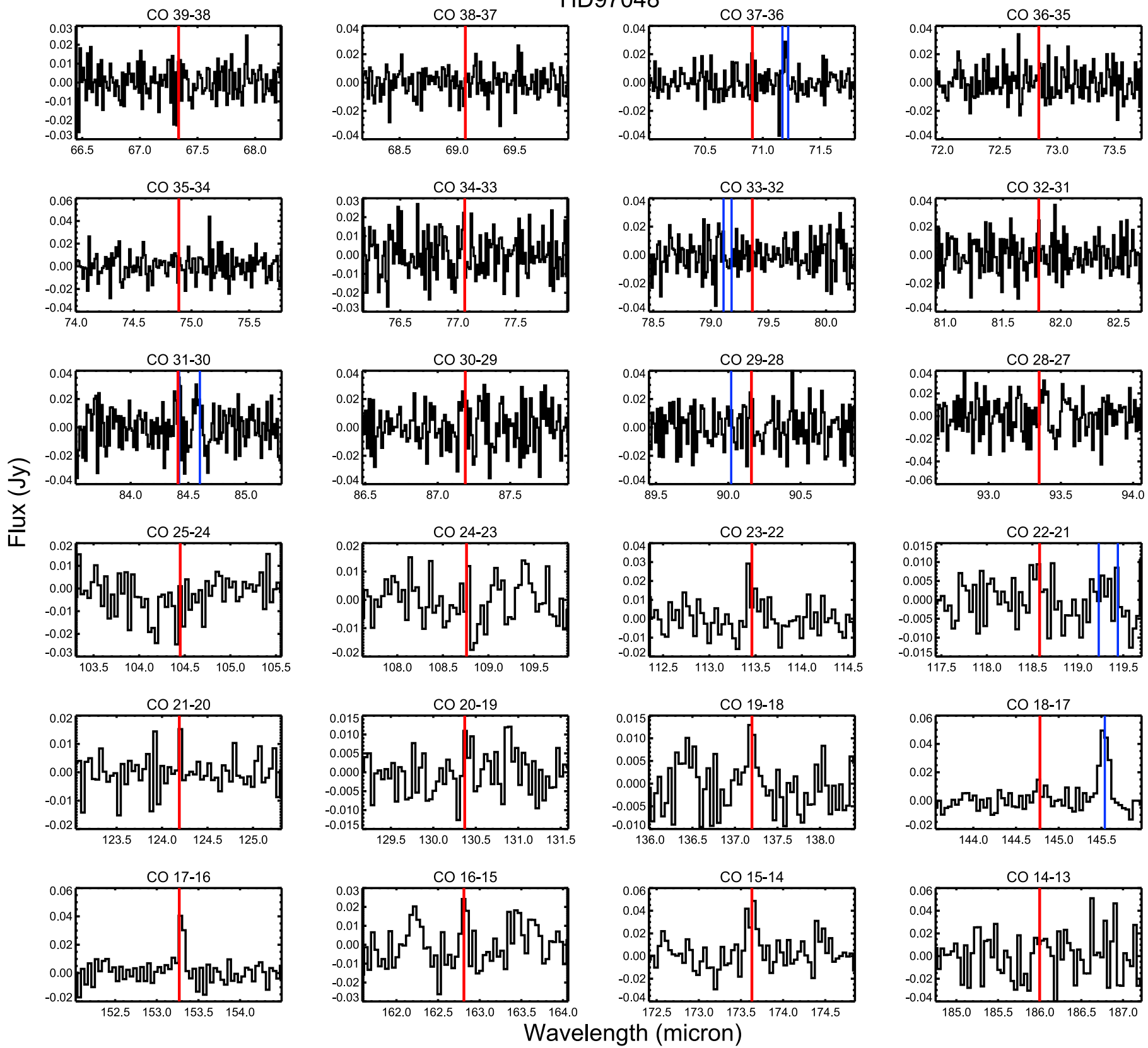

Fig. A.3. Spectra of HD 97048, centered on the $\mathrm{CO}$ lines, indicated in red. The $\mathrm{OH}$ line at $84.42 \mu \mathrm{m}$ blends with the $\mathrm{CO} J=31 \rightarrow 30$ line. In blue, we indicate the positions of other lines $\left(\mathrm{CH}^{+}\right.$at $90 \mu \mathrm{m}$ and $\left[\mathrm{O}_{\mathrm{I}}\right]$ at $145 \mu \mathrm{m}$; rest are $\mathrm{OH}$ doublets). 

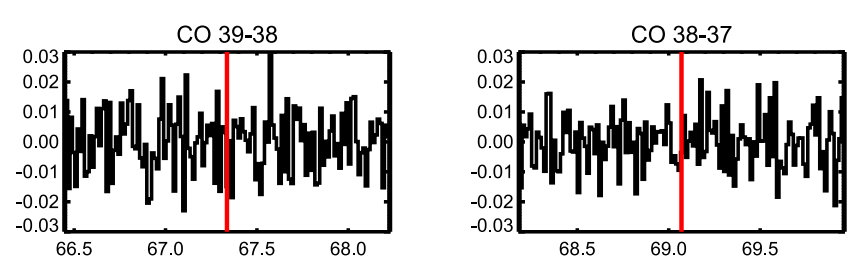

IRS48
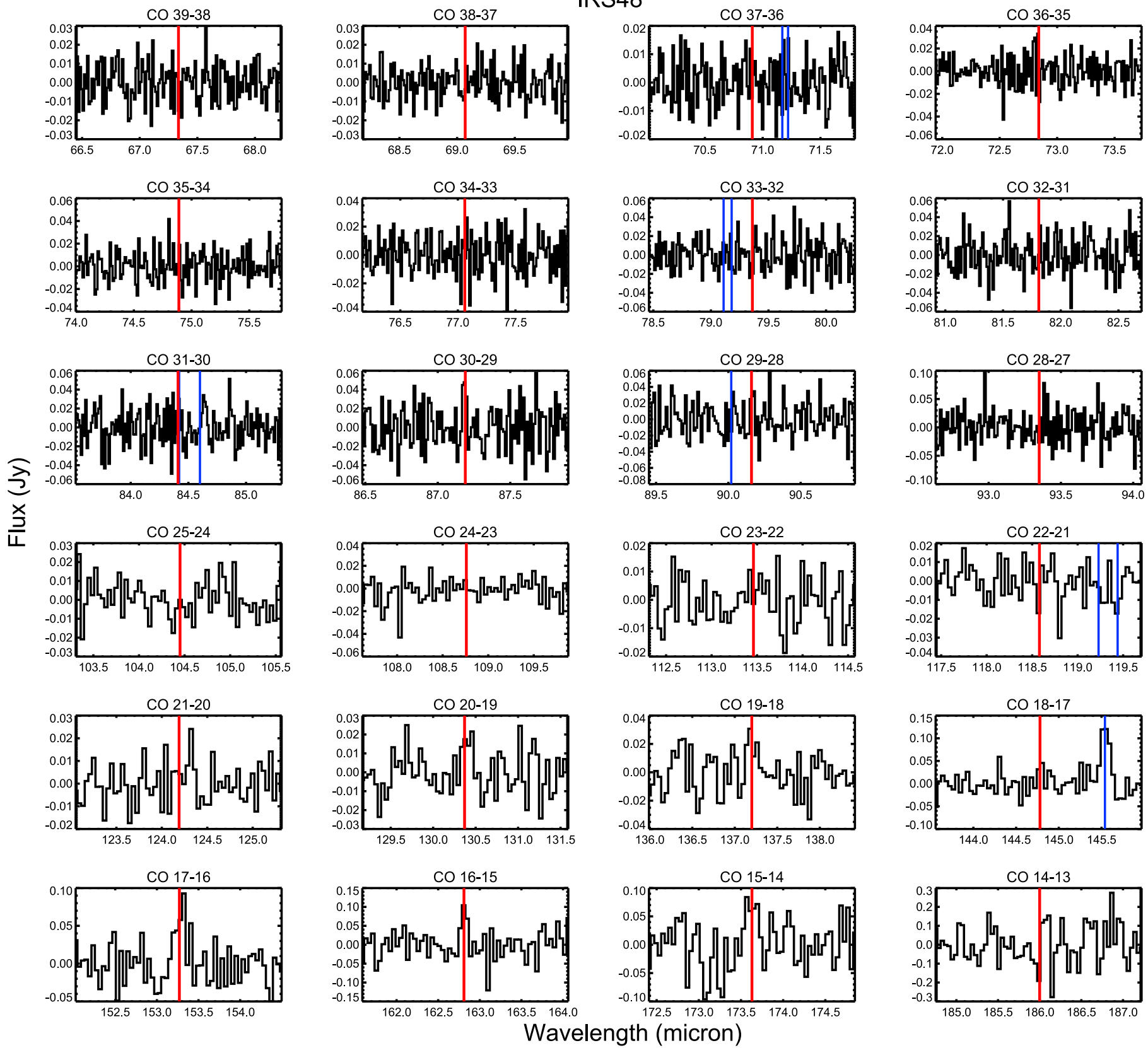

Fig. A.4. Spectra of IRS 48, centered on the CO lines, indicated in red. The OH line at $84.42 \mu \mathrm{m}$ blends with the CO $J=31 \rightarrow 30$ line. In blue, we indicate the positions of other lines $\left(\mathrm{CH}^{+}\right.$at $90 \mu \mathrm{m}$ and [O $\left.\mathrm{I}\right]$ at $145 \mu \mathrm{m}$; rest are $\mathrm{OH}$ doublets). 
G. Meeus et al.: $\mathrm{CO}$ in protoplanetary discs
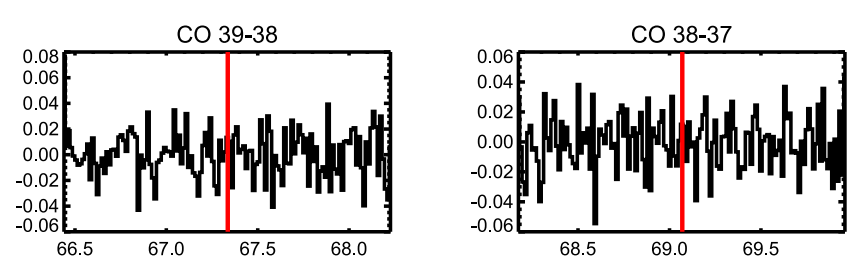

AS205
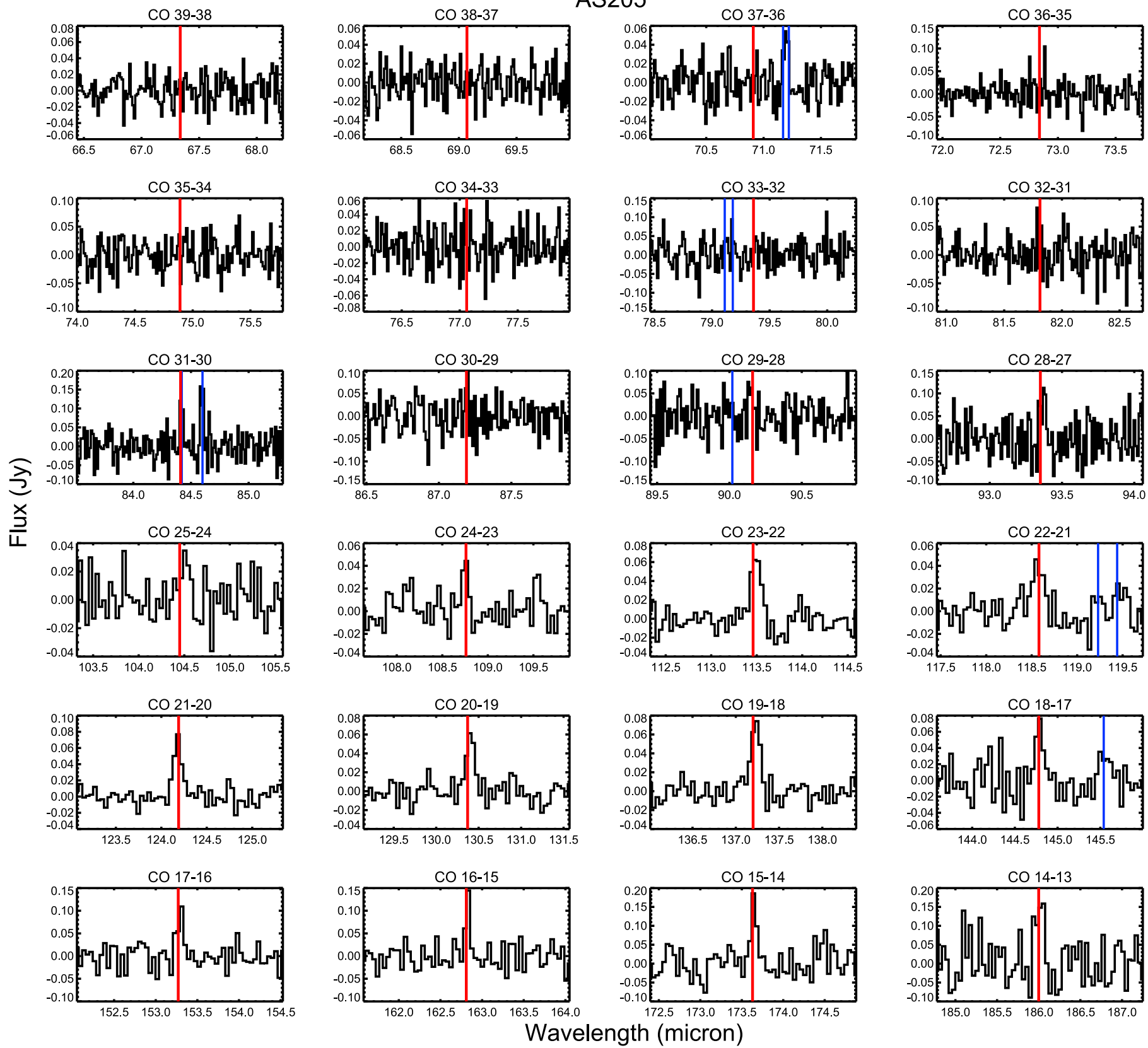

Fig. A.5. Spectra of AS 205, centered on the CO lines, indicated in red. The OH line at $84.42 \mu \mathrm{m}$ blends with the CO $J=31 \rightarrow 30$ line. In blue, we indicate the positions of other lines $\left(\mathrm{CH}^{+}\right.$at $90 \mu \mathrm{m}$ and $\left[\mathrm{O}_{\mathrm{I}}\right]$ at $145 \mu \mathrm{m}$; rest are $\mathrm{OH}$ doublets). 

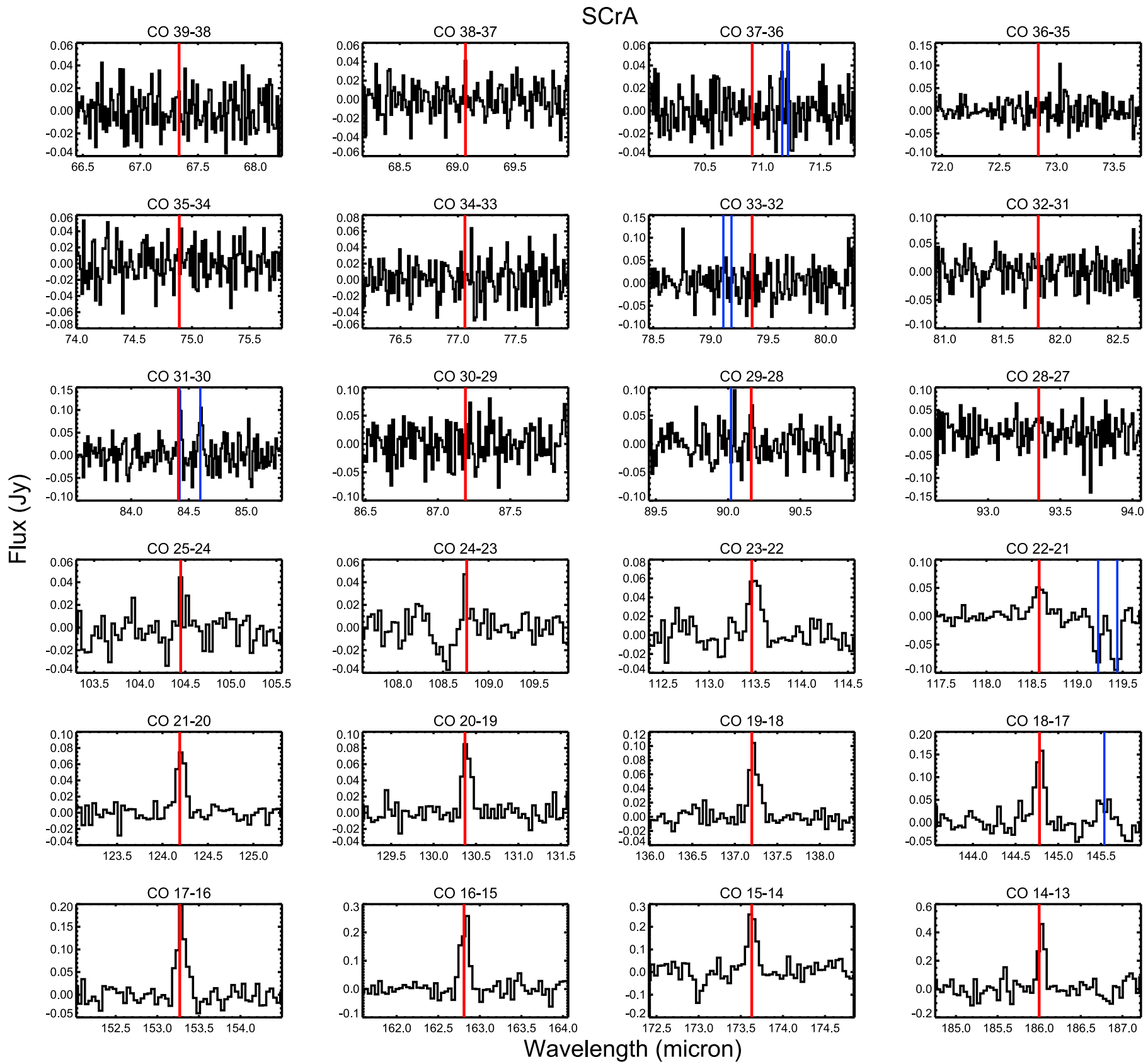

Fig. A.6. Spectra of S CrA, centered on the $\mathrm{CO}$ lines, indicated in red. The $\mathrm{OH}$ line at $84.42 \mu \mathrm{m}$ blends with the CO $J=31 \rightarrow 30$ line. In blue, we indicate the positions of other lines $\left(\mathrm{CH}^{+}\right.$at $90 \mu \mathrm{m}$ and $\left[\mathrm{O}_{\mathrm{I}}\right]$ at $145 \mu \mathrm{m}$; rest are $\mathrm{OH}$ doublets). 
G. Meeus et al.: $\mathrm{CO}$ in protoplanetary discs
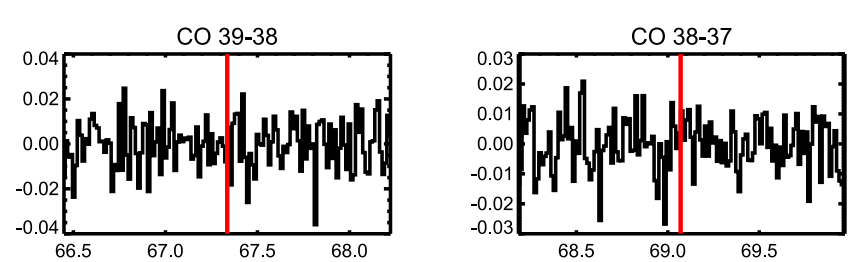

DGTau
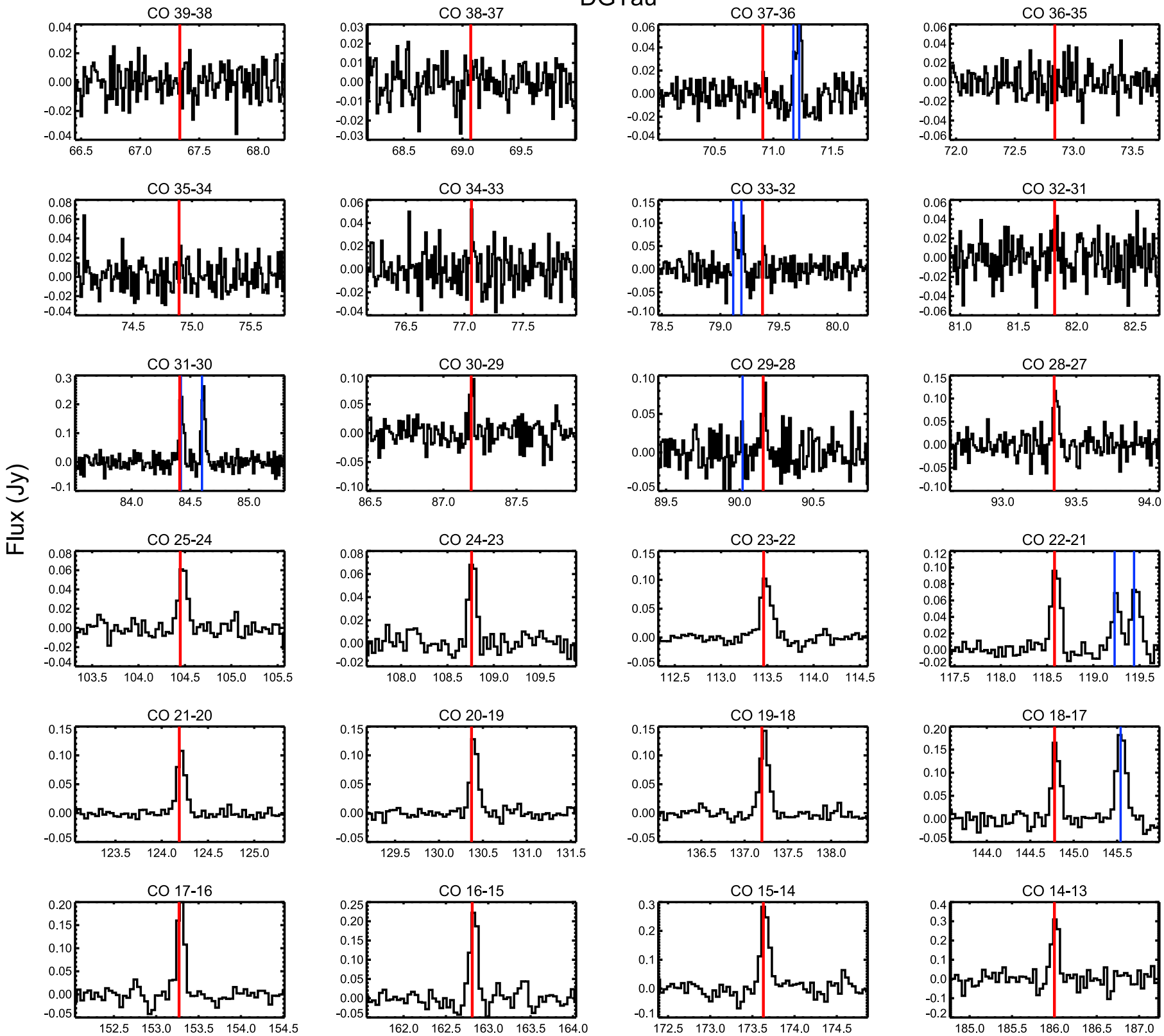

Fig. A.7. Spectra of DG Tau, centered on the CO lines, indicated in red. The $\mathrm{OH}$ line at $84.42 \mu \mathrm{m}$ blends with the CO $J=31 \rightarrow 30$ line. In blue, we indicate the positions of other lines $\left(\mathrm{CH}^{+}\right.$at $90 \mu \mathrm{m}$ and [O I] at $145 \mu \mathrm{m}$; rest are $\mathrm{OH}$ doublets).
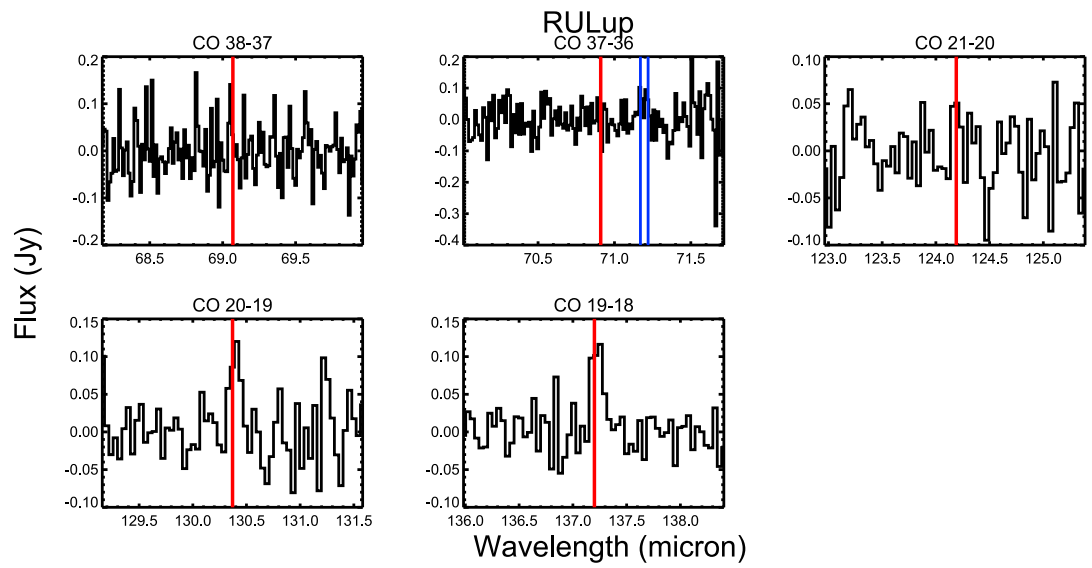

Fig. A.8. Spectra of RU Lup, centered on the CO lines, indicated in red. In blue, we indicate the position of OH lines. 


\section{Appendix B: Upper limits}

In Tables B.1 and B.2, we give the upper limits at a few key transitions $(J 36 \rightarrow 35,29 \rightarrow 28,23 \rightarrow 22,18 \rightarrow 17$, and $15 \rightarrow 14$ ) for those sources where not a single $\mathrm{CO}$ emission line was detected.

Table B.1. CO upper limits at selected wavelengths for the sources with full SEDs scans $(50-200 \mu \mathrm{m}) \mathrm{in} \mathrm{W} / \mathrm{m}^{2} \times 10^{-18}$.

\begin{tabular}{|c|c|c|c|c|c|c|c|c|c|c|}
\hline Object & $J 36 \rightarrow 35$ & $\begin{array}{c}\text { Cont. } \\
72.8 \mu \mathrm{m}\end{array}$ & $J 29 \rightarrow 28$ & $\begin{array}{c}\text { Cont. } \\
90.2 \mu \mathrm{m}\end{array}$ & $J 23 \rightarrow 22$ & $\begin{array}{c}\text { Cont. } \\
113.5 \mu \mathrm{m}\end{array}$ & $J 18 \rightarrow 17$ & $\begin{array}{c}\text { Cont. } \\
144.8 \mu \mathrm{m}\end{array}$ & $J 15 \rightarrow 14$ & $\begin{array}{c}\text { Cont. } \\
173.6 \mu \mathrm{m}\end{array}$ \\
\hline HD 35187 & $<5.7$ & 4.9 & - & - & $<16.7$ & 3.3 & $<10.8$ & 2.9 & - & - \\
\hline HD 38120 & $<20.2$ & 8.6 & $<25.5$ & 7.6 & $<11.9$ & 5.3 & $<9.6$ & 4.0 & $<13.7$ & 3.1 \\
\hline HD 50138 & $<33.0$ & 5.7 & $<33.6$ & 3.9 & $<12.9$ & 20.1 & $<12.7$ & 1.22 & $<17.1$ & 0.95 \\
\hline HD 100453 & $<67.1$ & 35.3 & $<20.7$ & 30.3 & $<14.5$ & 20.5 & $<9.8$ & 15.9 & $<19.1$ & 14.0 \\
\hline HD 104237 & $<53.9$ & 9.3 & $<27.6$ & 8.6 & $<13.9$ & 6.4 & $<11.7$ & 4.8 & $<19.0$ & 4.3 \\
\hline HD 135344 & $<24.6$ & 31.2 & $<19.1$ & 30.7 & $<12.8$ & 24.9 & $<6.9$ & 20.5 & $<15.5$ & 18.2 \\
\hline HD 139614 & $<32.8$ & 18.3 & $<21.7$ & 17.1 & $<11.2$ & 13.4 & $<9.5$ & 12.1 & $<17.2$ & 11.7 \\
\hline HD 142527 & $<7.6$ & 109.7 & $<37.2$ & 95.1 & $<29.6$ & 74.4 & $<22.1$ & 63.1 & $<37.9$ & 56.2 \\
\hline HD 144668 & $<36.8$ & 5.6 & $<21.5$ & 4.5 & $<13.4$ & 2.8 & $<8.2$ & 1.9 & $<20.1$ & 1.7 \\
\hline HD 163296 & $<4.1$ & 19.0 & $<23.9$ & 21.7 & $<13.6$ & 19.3 & $<11.8$ & 19.1 & $16.6(5.1)$ & 19.4 \\
\hline HD 169142 & $<121.3$ & 27.1 & $<53.5$ & 24.1 & $<33.0$ & 19.2 & $<20.4$ & 17.8 & $<20.1$ & 16.1 \\
\hline HD 179218 & $<22.8$ & 21.0 & $<13.9$ & 16.4 & $<10.1$ & 10.7 & $<6.5$ & 7.2 & $<12.2$ & 5.7 \\
\hline SR 21 & $<32.3$ & 33.5 & $<26.4$ & 29.9 & $<11.0$ & 21.8 & $<11.3$ & 16.6 & $<18.9$ & 13.9 \\
\hline
\end{tabular}

Notes. The continuum level is given in Jy.

Table B.2. CO upper limits for the sources with the forsterite scans $(60-71 \mu \mathrm{m}+120-143 \mu \mathrm{m}) \mathrm{in} \mathrm{W} / \mathrm{m}^{2} \times 10^{-18}$.

\begin{tabular}{lcccc}
\hline \hline Object & CO J38 $\rightarrow 37$ & Cont. $70.9 \mu \mathrm{m}$ & CO $J 21 \rightarrow 20$ & Cont. $124 \mu \mathrm{m}$ \\
\hline HD 98922 & $<30.8$ & 6.1 & $7.5:(3.2)$ & 1.1 \\
HD 141569 & $<22.6$ & 5.4 & $<13.1$ & 2.1 \\
HD 142666 & $<30.1$ & 6.7 & $<11.9$ & 5.1 \\
HD 144432 & $<16.7$ & 5.8 & $<8.0$ & 2.9 \\
HD 150193 & $<24.7$ & 7.2 & $<7.1$ & 3.2 \\
HT Lup & $<50.2$ & 6.2 & $<23.9$ & 11.0 \\
RU Lup & $<22.0$ & 5.9 & $<9.0$ & 4.2 \\
RY Lup & $<20.3$ & 6.3 & $<8.6$ & 3.9 \\
RNO90 & $<25.6$ & 3.8 & $<11.9$ & 2.1 \\
\hline
\end{tabular}

Notes. With “:” we indicate a detection that is between 2 and 3 sigma. The continuum level is given in Jy. 